\title{
Antennal Mechanosensory Neurons Mediate Wing Motor Reflexes in Flying Drosophila
}

\author{
Akira Mamiya and Michael H. Dickinson \\ Department of Biology, University of Washington, Seattle, Washington 98195
}

\begin{abstract}
Although many behavioral studies have shown the importance of antennal mechanosensation in various aspects of insect flight control, the identities of the mechanosensory neurons responsible for these functions are still unknown. One candidate is the Johnston's organ (J0) neurons that are located in the second antennal segment and detect phasic and tonic rotations of the third antennal segment relative to the second segment. To investigate how different classes of JO neurons respond to different types of antennal movement during flight, we combined 2-photon calcium imaging with a machine vision system to simultaneously record J0 neuron activity and the antennal movement from tethered flying fruit flies (Drosophila melanogaster). We found that most classes of J0 neurons respond strongly to antennal oscillation at the wing beat frequency, but not to the tonic deflections of the antennae. To study how flies use input from the J0 neurons during flight, we genetically ablated specific classes of J0 neurons and examined their effect on the wing motion. Tethered flies flying in the dark require JO neurons to generate slow antiphasic oscillation of the left and right wing stroke amplitudes. However, J0 neurons are not necessary for this antiphasic oscillation when visual feedback is available, indicating that there are multiple pathways for generating antiphasic movement of the wings. Collectively, our results are consistent with a model in which flying flies use JO neurons to detect increases in the wing-induced airflow and that $J 0$ neurons are involved in a response that decreases contralateral wing stoke amplitude.
\end{abstract}

Key words: antennal mechanosensory; Drosophila; flight control; machine vision; sensory motor integration; 2-photon imaging

\section{Introduction}

Flying insects must integrate sensory information from multiple modalities to control their flight (Taylor and Krapp, 2008). Although the neural circuits underlying visual processing have been studied extensively (Borst, 2014), much less is known about how flying insects acquire, process, and integrate other sensory channels. One of the sensory inputs essential for insect flight is antennal mechanosensation, which is thought to play an important role in the control of flight speed (Hollick, 1940; Heran, 1957; Gewecke, 1970; Gewecke et al., 1974; Niehaus, 1981), heading (Budick et al., 2007; Duistermars et al., 2009; Duistermars and Frye, 2010; Mamiya et al., 2011), and stabilization (Niehaus, 1981; Sane et al., 2007). Despite the extensive literature suggesting its importance, we do not know the identity of the mechanosensory neurons that are responsible for these functions. One candidate is the neurons of the Johnston's organ (JO) (Johnston, 1855), a large group of neurons that encode rotations of the third

Received Jan. 5, 2015; revised April 8, 2015; accepted April 13, 2015.

Author contributions: A.M. and M.H.D. designed research; A.M. performed research; A.M. analyzed data; A.M. and M.H.D. wrote the paper.

This work was supported by the Paul G. Allen Family Foundation to M.H.D. We thank Allan Wong for flies (J01, $J 0-A B$, JO-CE, and F GAL4 drivers, and JO-CE;eyeflp flies); Anne Sustar and Ainul Huda for technical assistance; and Bettina Schnell, Marie P. Suver, and Peter T. Weir for helpful discussions and comments.

The authors declare no competing financial interests.

Correspondence should be addressed to Dr. Michael H. Dickinson, Division of Biology and Bioengineering, California Institute of Technology, 1200 E. California Blvd, Pasadena, CA 91125. E-mail: flyman@caltech.edu.

M.H. Dickinson's present address: Division of Biology and Bioengineering, California Institute of Technology, 1200 E. California Blvd, Pasadena, CA 91125.

DOI:10.1523/JNEUROSCI.0034-15.2015

Copyright $\odot 2015$ the authors $\quad 0270-6474 / 15 / 357977-15 \$ 15.00 / 0$ antennal segment relative to the second segment (Eberl et al., 2000; Kamikouchi et al., 2009; Yorozu et al., 2009). In Drosophila, JO neurons can be classified into different classes (termed A-E) based on the location of their axon terminals in the antennal mechanosensory and motor center (AMMC) in the brain (Kamikouchi et al., 2006). In quiescent flies, JO-A, B neurons respond strongly to oscillatory movements of the antenna, such as those caused by courtship song, whereas JO-C, E neurons respond best to tonic deflections caused by gravity and wind (Kamikouchi et al., 2009; Yorozu et al., 2009). However, whether any of the JO neurons respond to antennal movements during flight is still unknown.

In a previous study, we showed that the antennae of tethered flying flies move both actively and passively during flight (Mamiya et al., 2011). Active movements are elicited by different types of large-field visual motion, whereas passive movements are caused by the airflow generated by the flapping wings. Passive movements can be further divided into small tonic deflections and rapid oscillations at wing beat frequency (Mamiya et al., 2011). In this study, we combined 2-photon calcium imaging and machine vision system to simultaneously monitor JO neuron activity and antennal motion in flying flies. Our goal was to investigate how different classes of JO neurons respond to different types of antennal movement. Our results show that most JO neurons respond strongly to antennal oscillation at wing beat frequency during flight, but not to tonic deflection of the antennae. To investigate the functional roles of JO neurons during flight, we genetically ablated specific classes of JO neurons and examined the effect on the wing motion of tethered flies flying in the dark. We found that JO neurons are involved in producing a slow 


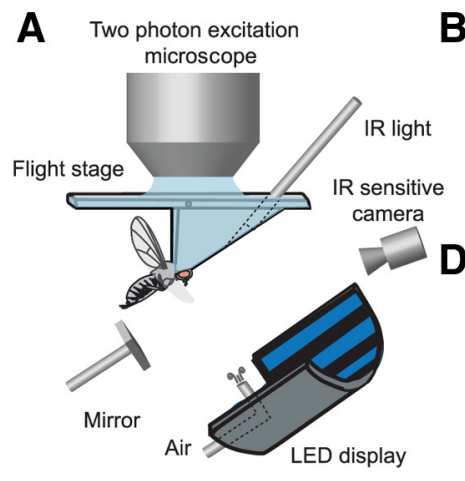

B

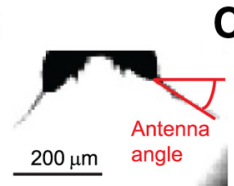

C

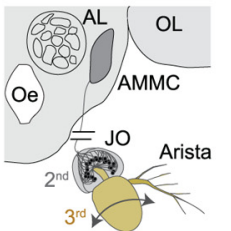

E

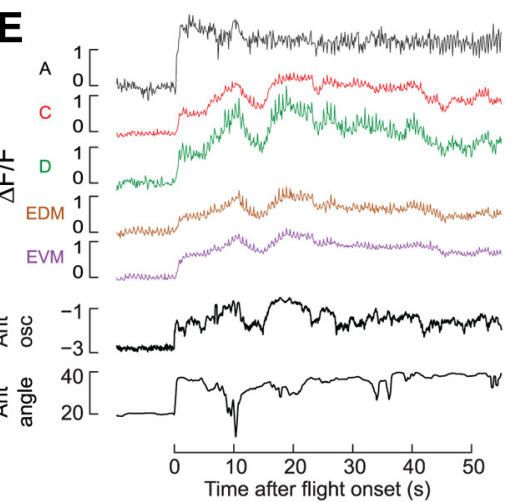

Figure 1. Simultaneously recording antennal motion and J0 neuron activity from tethered flying flies. $\boldsymbol{A}$, Imaging setup. Flies were tethered to a flight stage equipped with a light guide that delivers IR light to the fly's antennae. An IR-sensitive camera captured the backlit image of the aristae projected by a mirror. A 2-photon microscope recorded the calcium activity of the J0 neurons. A blue LED arena presented wide-field visual motions to the flies. Flight was initiated by delivering an air puff. $\boldsymbol{B}$, An image of the antennae captured by the IR-sensitive camera. We measured the angle between the arista and the horizontal plane of the image and defined it as the antenna angle. $\boldsymbol{C}$, A schematic of an antenna (third and second segment are shaded with light brown and light gray, respectively), J0 neurons (labeled J0), and their projection to the AMMC. J0 neurons have their cell bodies in the second antennal segment and respond to the rotation of the third antennal segment relative to the second antennal segment (indicated by a black arrow). Axons of the J0 neurons project to the AMMC in the brain. They can be categorized into different classes based on the location of their axon terminals in the AMMC. 0e, 0esopagus; AL, antennal lobe; OL, optic lobe. D, Left, A 3D reconstruction of the axon terminals of J0 neurons visualized by expressing GCaMP3 in these neurons using J01-GAL4. We classified axon bundles based on their morphology. Labels A-D indicates axon bundles of the J0-A (white), B (blue), C (red), and D (green) neurons, respectively. EDM and EVM indicate dorsal medial (brown) and ventral medial (magenta) branches of the JO-E neurons, respectively. Yellow region represents the main bundle of JO neuron axons that has not branched out yet. P, D, and M indicate posterior, dorsal, and medial direction, respectively. Right, An image of J0 neuron axons expressing GCaMP3 taken by a 2-photon microscope at a z-level where different classes of JO neurons form separate axonal branches. We classified the axons based on their morphology and manually selected the ROI for imaging calcium activities. A, C, D, EDM, and EVM indicate different classes of J0 neurons as in the left panel. Axon terminals of J0-B neurons cannot be seen in this image. $\boldsymbol{E}$, Top five traces, Example time courses of GCaMP3 fluorescence change relative to its baseline value $(\Delta \mathrm{F} / \mathrm{F})$ reflecting the calcium activity of $\mathrm{J} 0$ axon terminals shown in the right side of $\boldsymbol{D}$. Bottom two traces, The log ${ }_{10}$ of the antenna oscillation power at the wing beat frequency (top: unit $\operatorname{~deg}^{2} / \mathrm{Hz}$ ) and the mean antenna angle (bottom: unit degrees, calculated using 400 ms width sliding window) before and during flight. All the terminals increased their activity during flight, and the activity of JO-C, D, EDM, and EVM neurons during flight was correlated with the antenna oscillation power.

negative coupling between the left and right wing stroke amplitudes. Collectively, our results are consistent with a model in which flying flies use JO neurons to detect increases in the winginduced airflow and activate a response that decreases the contralateral wing stroke amplitude.

\section{Materials and Methods}

Experimental setup and preparation of flies. For all experiments, we used female Drosophila melanogaster 3-4 d after eclosion that were raised on a standard corn meal medium and kept at $25^{\circ} \mathrm{C}$ in a $14: 10 \mathrm{~h} \mathrm{light/dark}$ cycle. At the beginning of each experiment, we anesthetized flies using a Peltier stage held at $4^{\circ} \mathrm{C}$, and cut off their prothoracic and mesothoracic legs at the coxa-trochanter joint. We also cut off the tibia and tarsi of the metathoracic legs. In experiments in which we physically reduced antennal motion, we either cut off the aristae or glued the second and third antennal segments together with a UV-cured glue mixture composed of 4 parts of Watch Crystal Glue Clear Ultraviolet Adhesives (www. Esslinger.com) and 1 part of Loctite 3104 light cure adhesive (Henkel). Following previously published procedures (Maimon et al., 2010; Suver et al., 2012), we tethered the flies onto a flight stage that allows them to flap their wings freely while keeping their head stationary (Fig. $1 A$ ). We modified the flight stage to add a small light guide that provided IR illumination of the antennae (Fig. $1 A$ ) and recorded the antennal motion using an IR-sensitive camera (Basler A602f camera with an Infinistix 90 fixed-focus lens) (Fig. 1B). For behavioral experiments, we used the same camera to record images of the wing stroke envelope. Because the strong IR light necessary to track antennal motion interferes with the measurements of the wing stroke envelope, we were not able to record wing stroke amplitude simultaneously with antennal motion.

In imaging experiments, we recorded the activity of different JO neuron classes by imaging from the AMMC where the axon bundles of JO-A, B, C, D, E neurons separate into independent branches (Kamikouchi et al., 2006) (Fig. 1C,D). A previous anatomical study (Kamikouchi et al., 2006) has estimated the number of each type of JO neurons as follows: JO-A 159, JO-B 146, JO-C 31, JO-D 13, JO-E 71, and 58 cells project to multiple regions. The axon bundle of the JO-E neurons can be further divided into the dorsal medial (EDM) and ventral medial (EVM) branches (Fig. 1D). We covered the back of the fly's head with physiological saline (Olsen et al., 2007) and removed a piece of cuticle covering the posterior slope to expose the AMMC. We perfused saline (held at $\left.20^{\circ} \mathrm{C}\right)$ bubbled with carbogen $\left(95 \% \mathrm{O}_{2}, 5 \% \mathrm{CO}_{2}\right)$ at a rate of $2 \mathrm{ml} / \mathrm{min}$ throughout the experiment.

If a fly stopped flying during a trial, we immediately restarted flight by applying a brief air puff $(100 \mathrm{~ms}, 4.6 \mathrm{~m} / \mathrm{s})$ to the fly through a small plastic tube (ID: $3 \mathrm{~mm}$ ) located $30 \mathrm{~mm}$ below the fly head (Fig. $1 A$ ). To apply an air puff, we generated constant airflow with an air pump (Cole Parmer Instrument) connected to a flow controller (King Instrument) and briefly switched the flow toward the fly using a 3-way valve (Parker Hannifin). To exclude the effect of the air puff and to avoid segments when flies were not flying well, we removed the first and last $2 \mathrm{~s}$ of flight data from analysis whenever the flight was restarted.

Flies. To express the genetically encoded calcium indicator GCaMP3 (Tian et al., 2009) in different classes of JO neurons, we crossed flies carrying a transgene UAS-GCaMP3 with those carrying previously characterized GAL4 drivers, $J O 1, J O-A B$, and $J O-C E$, which drive expression in all JO neurons, JO-A/B neurons, and JO-C/E neurons, respectively (Kamikouchi et al., 2006). For control experiments, we crossed the flies carrying UAS-2xEGFP (Halfon et al., 2002) to those carrying the F-GAL4 driver that drives expression in all classes of JO neurons (Kim et al., 2003). We used the F-GAL4 driver for control experiments because the progeny of a cross between flies carrying JO1-GAL4 and UAS-2xEGFP did not fly well in our preparation.

We used a Canton-S strain (CS) as wild-type flies in behavioral experiments. For genetically ablating JO neurons using the protein synthesis inhibitor ricin A (Landel et al., 1988), we used an intersectional strategy that combines $J O-A B / J O-C E$ GAL4 driver with the eyeless-flippase (eyFLP) tissue-specific recombination system (Newsome et al., 2000). This was necessary because $J O-A B$ and JO-CE GAL4 drivers are expressed not only in JO neurons but also in neurons in the central brain. We crossed flies carrying UAS-stop-RicinA (UAS $\gg$ RicinA) (Bloomington stock \#29001) and UAS-2xEGFP with flies carrying JO-AB or JO-CE $G A L 4$ driver and $e y F L P$ and used the progeny for the experiment. In these flies, eyFLP is activated only in the eye and the antennae to remove the stop codon from UAS $\gg$ RicinA; therefore, ricin A is only expressed in 
the subset of these neurons that are driven by the JO-AB or JO-CE GAL4 driver. This intersectional strategy has been used successfully in a previous study to genetically ablate JO neurons (Yorozu et al., 2009). To further validate the selective expression of ricin A and the lack of leaky expression in individual flies, we inspected each fly's brain after the experiment using a 2-photon microscope and confirmed that EGFPlabeled JO neurons are not present, but EGFP signal could be detected in other central neurons driven by the JO-AB/JO-CE GAL4 driver. In these experiments, the progeny of wild-type (CS) flies crossed to the relevant parental strains (JO-AB-/JO-CE-GAL4;eyFLP, or UAS $\gg$ RicinA;UAS$2 x E G F P)$ served as controls.

Presentations of visual stimuli. We used a previously described light emitting diode (LED) display to present visual stimuli to the flies (Reiser and Dickinson, 2008). To separate the wavelength of the light emitted by the LED from the GCaMP3 fluorescence, we used blue LEDs (470 nm peak wavelength; Bright LED Electronics) and further reduced the longer wavelength component by covering the LEDs with three layers of blue gel filter (ROSCOLUX \#59 Indigo). The entire display consisted of 32 rows $\times 96$ columns of LED covering $32^{\circ}$ above and below the horizon and $108^{\circ}$ to the left and right of the midline. At the center of the display, each LED subtended a visual angle of $2.25^{\circ}$ on the fly's retina.

To induce different types of antennal motion during imaging experiments, we presented horizontal and vertical large-field motion to the flies by moving a square wave pattern with a fundamental spatial frequency of $36^{\circ}$ at the center ( $18^{\circ}$ of fully lit LEDs, $18^{\circ}$ of dark LEDs). The temporal frequency of the motion was set at $1 \mathrm{~Hz}$. Each presentation of the visual motion started with $2 \mathrm{~s}$ of static pattern presentation followed by $2 \mathrm{~s}$ of visual motion. In between the visual motion presentations, we presented an $18^{\circ}$ width dark stripe oscillating $\left(9^{\circ}\right.$ amplitude) at $1 \mathrm{~Hz}$ directly in front of the fly for $3 \mathrm{~s}$ to reset the bilateral balance of wing stroke amplitude. For each trial, we presented 8 visual motion stimuli in a pseudorandom order. In response to horizontal visual motion, flies actively move their antennae in a direction opposite to that of the visual motion (Mamiya et al., 2011). This increases the magnitude of the passive antennal oscillation for the antenna on the outside of a turn due to a decrease in the gap between the antennae and wing at ventral stroke reversal (Mamiya et al., 2011). At the same time, the antenna on the outside of the turn experiences a tonic passive deflection toward the ipsilateral wing (Mamiya et al., 2011). In response to upward visual motion, flies actively move their antennae forward and the magnitude of the antennal oscillation increases for both antennae (A.M., unpublished observation). The opposite movements occur in response to downward visual motion (A.M., unpublished observation). Because the magnitude of the response varied greatly among trials and flies, we used correlational analysis to determine which type of antennal motion activates the JO neurons.

To investigate whether flies with ablated JO-AB/JO-CE neurons can still generate bilaterally asymmetric changes of stroke amplitude in the presence of visual feedback, we presented a vertical square wave grating ( $36^{\circ}$ width; $18^{\circ}$ fully lit, $18^{\circ}$ dark) to the flies and allowed them to control the horizontal angular velocity of the pattern via a standard closed-loop paradigm using feedback through a machine vision system that tracked wing motion (Straw and Dickinson, 2009; Maimon et al., 2010). During visual closed-loop conditions with vertical stripes, flies tend to keep a vertical stripe centered in front of them by balancing the left and right wing stroke amplitudes (Reichardt and Wenking, 1969; Götz, 1987) and occasionally make rapid turns to fixate on a different vertical stripe. We also presented a static vertical grating and compared the wing movements under these conditions with those during a flight in the dark. Each condition lasted $6 \mathrm{~min}$, and the conditions were presented in a pseudorandom order.

Flight trials in the dark condition. To test the role of JO neurons in determining the phase relationship between the left and right wing angles, we investigated how the wing angles changed while tethered flies were flying in the dark condition. This condition was achieved by turning off the visual display and surrounding the setup with black curtains. Compared with the light intensity when the LED panels were on $(\sim 3$ $\mu \mathrm{W} / \mathrm{cm}^{2}$ ), the light intensity during the dark condition was at least 1000 time lower and below the measurement error of our photometer. Although we do not claim or need complete darkness, we refer to this condition as "dark" in our experiments because we were removing the light as much as possible. This condition allowed us to test the functional role of JO neurons in the absence of visual feedback. Each flight trial was 4-6 min long. To avoid strong bias in baseline wing position from affecting the data (as might be caused by asymmetry in tethering), we excluded flies that had absolute median left minus right wing stroke amplitude of $>25^{\circ}$ from the analysis ( 9 of 293 flies were excluded).

Tracking antennae and wing motion. To track the rotation of the third antennal segment relative to the second antennal segment, we acquired images of aristae $(100 \times 50$ pixels $)$ at 1000 frames $\sec ^{-1}$ using an IRsensitive camera (A602f; Basler) (Fig. 1A-C). To obtain a backlit image of the aristae, we made a small hole ( $1.5 \mathrm{~mm}$ diameter $)$ in the fly holder right above the head of the fly to fit a brass tube (ID $1.5 \mathrm{~mm}$ ) with a small light diffuser attached to the end. We illuminated the light diffuser by guiding light from a high intensity IR diode ( $880 \mathrm{~nm}$; Golden Dragon; Osram) to the diffuser using a plastic optical fiber (OD $1.5 \mathrm{~mm}$; Edmund Optics). The brass tube held the plastic optical fiber in place during the experiment. We projected the dark silhouette image of aristae (Fig. 1B) to the camera located in the front of the fly using a silver-coated mirror (Thorlabs) (Fig. 1A). To accurately measure the rotation of the third antennal segment relative to the second antennal segment, we tried to position the camera and the optical fiber at an angle that allows the imaging plane to be perpendicular to the rotational axis of the third antennal segment during flight. Because of physical constraints of the setup, our imaging plane was inclined $\sim 5^{\circ}$ to $8^{\circ}$ from the plane perpendicular to the rotational axis. Active movements of the antennae can slightly increase this mismatch between the imaging plane and the plane perpendicular to the rotational axis. However, because of the trigonometric relationships involved, this misalignment should cause errors of only $1 \%-2 \%$. To synchronize antenna images with 2-photon data, we saved the antenna images together with a signal that indicates the timing of 2-photon image acquisition using Motmot, open source Python software (Straw and Dickinson, 2009). Using a previously published algorithm (Mamiya et al., 2011), we calculated the angle of the aristae relative to the horizontal axis of the image and defined this as "antenna angle." Because the aristae are rigidly attached to the third antennal segment (Göpfert and Robert, 2001; Göpfert and Robert, 2002), tracking the aristae allowed us to measure the angle of the third antennal segment in the image plane. Based on the resolution of our images $(6.4 \mu \mathrm{m} / \mathrm{pixel})$ and the approximate length of a typical arista $(150 \mu \mathrm{m})$, one pixel movement at the tip of the arista corresponds to an antennal rotation of $\sim 2.5$ degrees. Because our arista tracking algorithm estimates the angle of the arista by taking an intensitythresholded image (which can be multiple pixels wide) and calculating the eigenvectors of the covariance matrix of this image (Mamiya et al., 2011), it is theoretically possible to achieve a resolution finer than one pixel movement at the tip of the arista. However, because our only validation of the tracking accuracy comes from visual inspections of the match between the calculated angle and the arista image, we do not have a quantitative estimate of this subpixel resolution accuracy. Thus, we conservatively estimate the angular resolution of our algorithm to be $\sim 2.5$ degrees. This is sufficient for measuring the large movements of the antennae during flight (Mamiya et al., 2011), but not sufficient for measuring smaller oscillatory movements of the antenna (such as those induced by courtship songs) that some JO neurons are known to respond to (Kamikouchi et al., 2009; Yorozu et al., 2009). For both the left and right antenna, we defined anterior rotations as positive changes in the antenna angle. To monitor wing angles during behavioral experiments, we illuminated the flies from the posterior side using an IR LED ( $850 \mathrm{~nm}$; Thorlabs) and captured the images of the wing stroke envelope at 100 frames $\mathrm{sec}^{-1}$ using an IR-sensitive camera (A602f; Basler). Using a previously described plug-in for Motmot (Maimon et al., 2010), we calculated the angle between the ventral reversal point of the wing stroke envelope and the horizontal axis of the image in real time and defined this as the "wing angle." For both the left and right wings, we defined anterior rotations as positive changes in wing angle.

Spectral analysis of antennae and wing angles. We used multitaper spectral estimation methods implemented in the Chronux toolbox (http://www.chronux.org/) for MATLAB (MathWorks) (Mitra and Bokil, 2008) to estimate the antenna and wing oscillation power, and the 
coherence and phase lag between the left and right wing angles. For estimation of antennal oscillation power, we used a $0.4 \mathrm{~s}$ time window, 5 $\mathrm{Hz}$ bandwidth, and three tapers. We moved the window in $50 \mathrm{~ms}$ steps to estimate how antennal oscillation power changed throughout the trial. We also used the same $0.4 \mathrm{~s}$ moving window to calculate the mean antenna angle during flight. The antennal oscillation power has a narrow peak at the wing beat frequency (Mamiya et al., 2011); therefore, we used the maximum power between 150 and $250 \mathrm{~Hz}$ (a band that encompasses wingbeat frequency) in each window as the antennal oscillation power for that time point. These parameters for the spectral analysis and power extraction have been used successfully in a previous study to estimate antennal oscillation power at wing beat frequency (Mamiya et al., 2011). For spectral analysis of wing angles, we first divided the entire flight sequence into $4 \mathrm{~s}$ segments and used a $4 \mathrm{~s}$ time window, $0.5 \mathrm{~Hz}$ bandwidth, and three tapers to calculate the power, coherence, and phase lag for each segment. The coherence between the left and right wing angles measures the stability of the phase relationship and was calculated as follows:

$$
a b s\left(\frac{S_{\text {left,right }}(f)}{\sqrt{S_{\text {left }}(f) S_{\text {right }}(f)}}\right)
$$

where $S_{\text {left,right }}(f)$ is the cross-spectrum between the left and right wing angles and $S_{\text {left }}(f)$ and $S_{\text {right }}(f)$ are the individual spectra of the left and right wing angles, respectively. We averaged these values over the entire flight trial to obtain the estimates for each fly. To avoid using unstable flight segments, we excluded flights that were $<5$ s from further analysis. For all analysis of antenna and wing oscillation power, we used $\log _{10}$ of the power to better approximate a normal distribution.

Image acquisition and analysis. We imaged JO neuron activity from tethered flying flies using the Ultima IV (Prairie Technologies) 2-photon microscope with a mode-locked Ti:Sapphire laser (Chameleon Ultra; Coherent) tuned to $930 \mathrm{~nm}$. We adjusted the laser power to be $<20 \mathrm{~mW}$ at the rear aperture of the objective lens (Nikon NIR Apo, $40 \times$ waterimmersion lens, 0.8 NA). We bandpass filtered GCaMP/EGFP fluorescence with HQ525/50 m-2p emission filter (Chroma Technologies) and collected the photons using a multialkali photomultiplier tube (Hamamatsu). To simultaneously record activities of different JO neuron classes, we imaged at a $z$-level where axon bundles of JO-A, B, C, D, EDM, and EVM neurons separate into independent branches (Kamikouchi et al., 2006) (Fig. 1C,D). For each trial, we acquired 650 images $\left(152 \times 150\right.$ pixels, $0.125 \mathrm{~s}$ frame ${ }^{-1}, 81.25 \mathrm{~s}$ total $)$ while presenting widefield visual motion to induce changes in wing stroke amplitude and consequent changes in antennal movement (Mamiya et al., 2011). We recorded 2-4 trials at each $z$-level and repeated the experiment at 2-4 different $z$ levels that were $10 \mu \mathrm{m}$ apart. We did not see any significant differences in the JO neuron responses at the range of the $z$ levels we tested (flight response, $p>0.05$, Kruskal-Wallis nonparametric one-way ANOVA; partial correlations between JO activity and antennal motions, $p>0.05$, bootstrap analysis). Thus, we grouped all recordings from a fly together for analyses. At the end of each experiment, we acquired a $z$-series ( $1 \mu \mathrm{m}$ step) of JO axonal branches to confirm the location of each recording and to classify JO neuron types.

We analyzed images using a custom program written in MATLAB (MathWorks). We first smoothed the images with a Gaussian filter $(3 \times$ 3 pixel, $\sigma=0.5$ ) and corrected for small $x-y$ brain movements using a previously published algorithm that registers images using matrixmultiply discrete Fourier transform (Guizar-Sicairos et al., 2008). After the image registration, we classified JO neuron terminals according to their projection pattern (Kamikouchi et al., 2006) and manually selected an ROI for each JO neuron class. We treated all pixels in a ROI as a functionally homogeneous group because we did not see clear difference in the responses of pixels within each ROI, and previous imaging studies have not found any evidence for functional heterogeneity within each class of JO neurons (Kamikouchi et al., 2009; Yorozu et al., 2009). However, there is still a possibility that there are some functional differences among individual JO neurons classified as a single class and that these differences were not detectable with the spatial-temporal resolution of our imaging method. To account for the noise and the light leakage from the LED panel, we also manually selected a region with no clear fluorescence signal and defined the mean intensity of that region as the background. For each frame, we calculated the mean fluorescence signal of each ROI (F) by subtracting the background from the mean ROI pixel intensity. At each $z$-level, we calculated the F during the quiescent state $\left(\mathrm{F}_{0}\right)$ for each ROI by taking the average ROI pixel intensity during the 4 frames before the onset of the flight. We used the change in ROI fluorescence signal relative to its baseline $\left(\left(\mathrm{F}-\mathrm{F}_{0}\right) / \mathrm{F}_{0}\right)$ as a measure of JO neuron activity, and abbreviated this as $\Delta \mathrm{F} / \mathrm{F}$. For each fly, we calculated the mean flight response of each JO neuron class by averaging all the $\Delta \mathrm{F} / \mathrm{F}$ values of the corresponding ROI during flight. We only used segments where flies were continuously flying for $>30 \mathrm{~s}$.

Several factors other than the neural activity could affect the absolute magnitude of the $\Delta \mathrm{F} / \mathrm{F}$ signals in the different ROIs. Such factors include the extent to which the neural activity increases the intracellular calcium and the density of axons in our ROIs. Because these factors could vary with cell type, the differences in the magnitude of the $\Delta \mathrm{F} / \mathrm{F}$ signals we recorded may not accurately predict differences in spike rates across JO neuron classes.

Calculating partial correlations between the $\Delta F / F$ and the antennal motion. To determine what type of antennal motion activates the JO neurons during flight, we calculated the correlation between each JO neuron class activity and the antennal oscillation power or the mean antenna angle. Because the antennal oscillation power and mean antenna angle were estimated using a sliding window (400 ms width, $50 \mathrm{~ms}$ slide length; see Spectral analysis of antennae and wing angles), we also calculated sliding window averages of $\Delta \mathrm{F} / \mathrm{F}$ using the same parameters. Before calculating correlations, we convolved the mean antenna angle and the $\log _{10}$ of antennal oscillation power with exponentially decaying kernels to account for the exponential decay of the GCaMP3 fluorescence in response to an instantaneous drop in the intracellular calcium level. Because the time constant of the decay has been suggested to be $\sim 500 \mathrm{~ms}$ (Sun et al., 2013), we chose three different kernels with time constants of 250,500 , and $1000 \mathrm{~ms}$. The amplitudes of the kernels were adjusted so that each kernel had the same area, which allowed us to compare the time course of the convolved signals without the effect of the temporal accumulation of the signal. Because changes in the antennal oscillation power and the mean antenna angle could themselves be correlated with each other during flight, we calculated partial correlations between the $\Delta \mathrm{F} / \mathrm{F}$ and each type of antennal motion while accounting for the effect of the other type of motion. The partial correlation between variables $x$ and $y$ accounting for the effect of a variable $z\left(\rho_{\mathrm{xy} \cdot z}\right)$ was calculated using the following equation:

$$
\rho_{x y} \bullet z=\frac{\rho_{x y}-\rho_{x z} \rho_{z y}}{\sqrt{1-\rho_{x z}^{2}} \sqrt{1-\rho_{z y}^{2}}}
$$

where $\rho_{\mathrm{xy}}$ represents the correlation coefficient between variables $x$ and $y$ (Yule, 1896). These partial correlations between the GCaMP signal and each type of antennal motion convolved with a GCaMP kernel are likely to be an underestimate of the actual partial correlations between antennal motion and JO neuron activity. We suspect that there are nonlinear processes and additional temporal dynamics involved in converting JO neuron activity into the intracellular free calcium concentration that obscure the actual correlation. As in the calculation of the flight response, we only used segments in which flies were continuously flying for $>30 \mathrm{~s}$. When averaging the partial correlation values across the trials for each fly, we first converted the partial correlation values to $z$-values using the Fisher $z$-transformation, and after calculating the mean $z$-value, transformed it back to the partial correlation values.

One possible complication for using the partial correlation between JO neuron activity and mean antenna angle to study how JO neurons respond to tonic changes in the antenna angle is that both the active movement and the passive deflection of the antenna contribute to the changes in the mean antenna angle during flight (Mamiya et al., 2011). Although it is possible to separate these two effects by physically inhibiting the active movements of the antennae, we chose not to do so because we wanted to study the responses of JO neurons to realistic movements of the antennae during flight. Because active movements of the antenna 
may sometimes mask the passive antennal deflections, we consider the partial correlations with the mean antenna angle to be a conservative estimate of the actual correlations between the mean antenna angle and JO neuron activity. To further verify our results, we also calculated the correlation between the activity of the JO-C and E neurons during flight. JO-C neurons are excited by tonic anterior rotation and slightly inhibited by tonic posterior rotation whereas JO-E neurons exhibit the opposite response (Yorozu et al., 2009). Thus, if these two neuron classes respond to tonic changes in antenna angle, then their activity should be negatively correlated during flight. To be able to detect the negative correlations between the JO-C and JO-E neurons, even when they are both positively correlated with antennal oscillation power, we calculated partial correlations between their activities accounting for the changes in antennal oscillation power.

Our correlation analysis would fail if flies did not change their antennal oscillation power and mean antenna angle during a trial. Therefore, we excluded trials in which the antennal oscillation power and the mean antenna angle did not change at least 10 -fold and $5^{\circ}$, respectively. These criteria were set based on previously published results on the changes in antenna oscillation power and mean antenna angle in tethered flying flies responding to wide-field visual motion (Mamiya et al., 2011). Approximately $90 \%$ of all trials met these criteria. For consistency, trials that did not meet the above criteria were also excluded from the calculation of the mean flight response of JO neurons.

Statistical tests. We used the statistics toolbox in MATLAB for all statistical analyses. We used nonparametric statistical tests when analyzing flight responses because the distributions of these values were significantly different from normal distribution ( $p<0.05$, Lilliefors' composite goodness-of-fit test). When comparing two groups of partial correlation values, we first converted them to $z$-values and used bootstrap analysis (10,000 samples) to estimate the distribution of the mean difference between the two groups. We used this distribution to test whether the differences between the partial correlation values were statistically significant. When testing the significance of the partial correlation values, rather than testing whether these values were significantly different from zero, we tested whether the partial correlation values between the GCaMP signal and the antennal motion were significantly different from those between the EGFP signal and the antennal motion. This is the appropriate comparison to test against the possible artifact caused by brain motion during steering maneuvers. For these tests, we also used the same Fisher $z$-transformation and bootstrap analysis mentioned above. Whenever there were multiple planned comparisons, we adjusted for multiple comparisons using Bonferroni correction.

For behavioral experiments, we calculated the $95 \%$ confidence intervals of the left and right wing oscillation power, coherence, and phase lag using bootstrap method (10,000 samples). We also used bootstrap method (10,000 samples) to test whether the physical manipulations of the antennae or the visual feedback significantly changed the antiphase oscillation between the left and right wing angles in the specific frequency band. In these tests, we used a two-tailed test to inspect whether the physical manipulations or the visual feedback significantly changed the horizontal component of the mean difference vectors.

\section{Results}

JO neuron classes show different levels of GCaMP signal in response to ipsilateral antennal motion during flight

To investigate how JO neurons respond to antennal motion during flight, we expressed the genetically encoded calcium indicator GCaMP3 (Tian et al., 2009) in different JO neuron classes and recorded their activity using 2-photon microscopy while simultaneously tracking antennal motion (Fig. $1 A-D$ ).

The top five traces in Figure 1E show an example of calcium activity in the axon terminals of different JO neuron classes during flight. The trace second from the bottom shows the power of antennal oscillation at wing beat frequency (shown as the $\log _{10}$ of the power), and the bottom trace shows the mean antenna angle (calculated using $400 \mathrm{~ms}$ boxcar filter) (Fig. 1E). As can be seen in this example, JO neurons increase their activity at the onset of flight. Furthermore, this activity fluctuates during flight as the power of antennal oscillation and the mean antenna angle change. The $\Delta \mathrm{F} / \mathrm{F}$ response (the change in the GCaMP fluorescence intensity relative to its baseline value) varies, however, among the ROIs containing different classes of JO neurons. We calculated the average $\Delta \mathrm{F} / \mathrm{F}$ during the entire flight bout for each JO neuron class in each fly (Fig. 2, leftmost columns for each JO neuron class). $\Delta \mathrm{F} / \mathrm{F}$ signal in ROIs containing JO-A and JO-C neurons increased the most during flight, whereas the changes in ROIs containing JO-B and JO-D neurons were more moderate (Fig. 2, left side of the black dotted line). We observed a similar pattern when we expressed GCaMP3 in specific subsets of cells using GAL4 drivers with more limited expression (JO-AB-GAL4 and JO-CE-GAL4; Fig. 2, right side of the black dotted line), suggesting that these differences in the $\Delta \mathrm{F} / \mathrm{F}$ responses were not due to a misclassification of JO neurons based on ROIs. EGFP fluorescence in control flies did not change significantly, indicating that the measured increases in GCaMP fluorescence during flight were not due to brain motion (Fig. 2, middle columns for each JO neuron type, $p>0.05$, Wilcoxon signed rank test with Bonferroni correction). The stronger $\Delta \mathrm{F} / \mathrm{F}$ signals in JO-A and JO-C neurons might indicate intrinsic differences in sensitivity among JO neuron classes. However, the results might also be due to other factors, such as variation in the processes linking spike rate to intracellular calcium accumulation in terminals or differences in baseline activity (see Materials and Methods). Among the axons of JO-E neurons, $\Delta \mathrm{F} / \mathrm{F}$ in EVM regions increased significantly more during flight compared with those in EDM regions $(p<0.05$, Tukey-Kramer post hoc comparison of the medians) and for this reason we analyzed these branches separately in all further experiments.

Previous studies have shown that all JO neurons project ipsilaterally, except for a very small subgroup of JO-E neurons that project to the EDC region (Kamikouchi et al., 2006). Therefore, if the increase in the activity during flight was due to the movements of the antennae, then physically restricting the ipsilateral antenna should largely eliminate this response. On the other hand, if the calcium response in JO terminals is due to centrifugal inputs, then blocking the movements of the ipsilateral antenna may not eliminate the increase. To distinguish between these two possibilities, we restricted the rotation of the third antennal segment relative to the second antennal segment using UV-activated glue and imaged JO neurons that were ipsilateral to the glued antenna during flight (Fig. 2, right most columns for each JO neuron type). In all JO neurons, except EDM neurons, which do not show an increase in activity during flight, gluing the ipsilateral antenna abolished the flight response $(p<0.01$, TukeyKramer post hoc comparison of the medians). Thus, the measured responses in JO neurons during flight are most likely caused by mechanosensory activation induced by motion of the ipsilateral antenna.

Although there was a clear difference in the average strength of the flight response among the different classes of JO neurons, there was also large fly-to-fly variability. We determined that this variability was not due to inconsistencies with imaging depth (see Materials and Methods). To test whether the variability was due to differences in the magnitude of antennal movement in different flies, we calculated the correlation between the strength of flight response and the mean antenna angle or the average power of oscillation at wing beat frequency. We found no significant correlation for either parameter ( $p>0.05$, Pearson's correlation coefficient with Bonferroni correction). Thus, we cannot explain the interfly variability, which might be due to a number of factors, 
including GCaMP expression strength or the behavioral state of the animal during flight.

The activities of JO-C, D, EDM, and EVM neurons are highly correlated with the power of antennal oscillation The results from experiments in which we glued the antennae suggest that the activity of JO neurons is due to movement of the ipsilateral antenna. We tested whether the responses were due to tonic or oscillatory movement by calculating the correlation between the measured GCaMP responses and the changes in either the mean antennal angle or the power of antennal oscillation at wing beat frequency (Fig. 3). Because the two parameters might be correlated with one another, we calculated partial correlations between each type of antennal motion and JO neuron activity, which accounted for the effect of the other type of motion. To account for the time it takes for the GCaMP fluorescence to decay after an instantaneous drop in intracellular calcium, we convolved both the power of antenna oscillation at wing beat frequency and the mean antenna angle with kernels that exponentially decay with time constants based on previous experiments (Fig. 3A) (Sun et al., 2013). In the range of values tested, we found no significant difference between the partial correlations calculated using different decay time constants ( $p>0.05$, bootstrap analysis). Thus, we show only the partial correlations to the traces convolved using the kernel with a $500 \mathrm{~ms}$ time constant, which is closest to the experimentally measured value for the time constant of GCaMP3 (Sun et al., 2013).

Changes in the activity of JO-C, D, EDM, and EVM neurons during flight were all strongly correlated with oscillation power at wing beat frequency (Fig. 3B, left side of the blue dotted line; $p<$ 0.01 compared with EGFP controls, bootstrap analysis with Bonferroni correction). The activity of JO-B neurons also correlated with the oscillation power, but this relationship was significantly smaller than those observed in the above groups ( $p<0.05$ compared with JO-C, D, EDM, and EVM, bootstrap analysis with Bonferroni correction). Although JO-A neurons showed strong activity during flight (Fig. 2), their activity was not correlated with oscillation power ( $p>0.05$ compared with EGFP controls, bootstrap analysis with Bonferroni correction), suggesting that their response may be saturated during flight. EDM neurons, which did not exhibit an increase in overall activity during flight, nevertheless exhibited changes in the activity that were highly correlated with oscillation power.

In contrast to the high correlation with oscillation power, the activity of most JO neurons did not show strong correlations with changes in the mean antenna angle during flight (Fig. 3B, right side of the blue dotted line). Activity of JO-A, D, EDM, and EVM neurons was not significantly correlated with mean antenna an- gle ( $p>0.05$ compared with EGFP controls, bootstrap analysis with Bonferroni correction). The activity of JO-B and JO-C neurons did show significant correlations with mean antenna angle $(p<0.01$ compared with EGFP controls, bootstrap analysis with Bonferroni correction), but for JO-C neurons, the correlations were not as strong as they were with oscillation power $(p<0.01$, bootstrap analysis with Bonferroni correction). To further confirm that JO-E neurons were not responding to tonic changes in antenna angle, we also calculated the correlation between the activity of JO-C neurons and those of EDM or EVM neurons during flight. JO-C neurons are known to respond to tonic anterior rotations of the antenna, whereas JO-E neurons respond to tonic posterior rotations (Kamikouchi et al., 2009; Yorozu et al., 2009). Therefore, if JO-C and JO-E neurons were responding to tonic changes in antenna angle, their activity should be negatively correlated. However, even after accounting for the correlation with oscillation power, we did not find significant negative correlation between the activities of these neurons (data not shown; $p>0.05$, Pearson's correlation coefficient with Bonferroni correction), further confirming that JO-E neurons are not responding to tonic changes in antenna angle. 

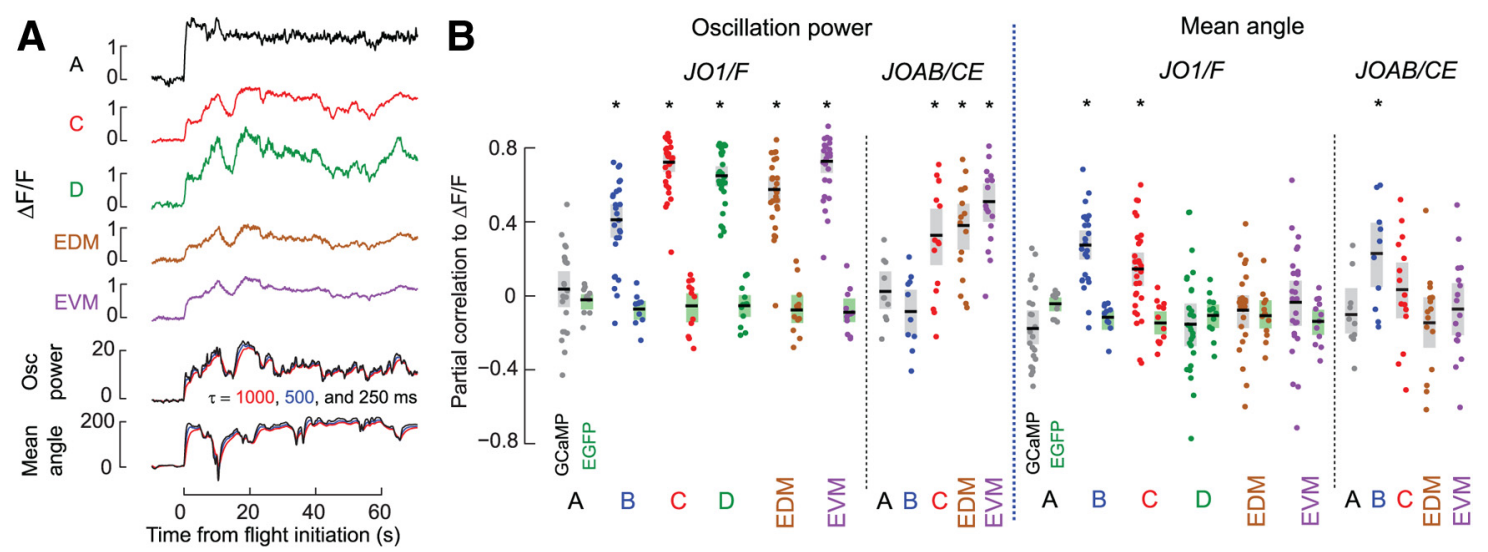

Figure 3. Activities of $J 0-C, D$, and Eneurons during flight are highly correlated with antennal oscillation power. $A$, Top five traces, An example time course of GCaMP3 fluorescence change relative to baseline $(\Delta F / F)$ in axon terminals of $J 0-A, C, D, E D M$, and EVM neurons. These traces are the same as those shown in Figure $1 E$, except that they have been temporally filtered with the same sliding window ( $400 \mathrm{~ms}$ width, $50 \mathrm{~ms}$ sliding length) used during the calculation of the antenna oscillation power and the mean antenna angle. Bottom two traces, The same $\log _{10}$ of the antenna 0 scillation power (second from the bottom, unit: $\mathrm{deg}^{2} / \mathrm{Hz}$ ) and the mean antenna angle (bottom, unit: degrees) shown in Figure $1 E$, but convolved by exponentially decaying GCaMP3 kernels with time constants of 1000 (red lines), 500 (blue lines), or 250 (black lines) ms to account for the time it takes for the GCaMP fluorescence to decay after an instantaneous drop in intracellular calcium. Activities of JO-C, D, EDM, and EVM neurons, but not JO-A neurons, correlated well with the antennal oscillation power during flight. $\boldsymbol{B}$, Left of the blue dotted line, Partial correlations between the antenna oscillation power convolved by a GCaMP3 kernel $(\tau=500 \mathrm{~ms}$ ) and the $\Delta \mathrm{F} / \mathrm{F}$ of GCaMP3 (left columns for each J0 neuron class), or EGFP (right columns for each J0 neuron class) expressed in different $\mathrm{J} 0$ neuron classes. Circles represent the mean partial correlation calculated for the entire flight trials for each J0 neuron class in one fly. Gray and green shaded boxes represent the $95 \%$ confidence intervals of the partial correlations with the $\triangle \mathrm{F} / \mathrm{F}$ of GCaMP3 and EGFP, respectively. Black horizontal lines indicate mean values. Panel on the left of the black dotted vertical line, Results from experiments using $J 01$ or F-GAL4 drivers that drive expression in all JO neurons. Panels on the right, Results from experiments using more specific JO-AB-GAL4 or JO-CE-GAL4 drivers. Asterisks indicate partial correlations that are significantly different from those for EGFP controls: ${ }^{*} p<0.01$ (bootstrap analysis with Bonferroni correction). Number of flies for each experiment is the same as those shown in Figure 2. Right of the blue dotted line, Same as the left side, except that it shows partial correlations with the mean antenna angle convolved with a GCaMP3 kernel ( $\tau=500 \mathrm{~ms}$ ).

To independently verify the responses within different classes of JO neurons, we expressed GCaMP3 in subsets of JO neurons using JO-AB-GAL4 and JO-CE-GAL4 drivers and repeated the experiments (Fig. $3 B$, right side of the black dotted lines). The results from these experiments confirmed that the changes in the activity of JO-C, EDM, and EVM neurons are strongly correlated with the oscillation power $(p<0.01$ compared with EGFP controls, bootstrap analysis with Bonferroni correction). As with the experiments using JO1-GAL4 driver, the activity within JO-A neurons showed no significant correlation with either oscillation power or mean antenna angle ( $p>0.05$ compared with EGFP controls, bootstrap analysis with Bonferroni correction). Although the results using general and more specific GAl4 drivers were similar, we did find two differences. In experiments using the JO1-GAL4 driver, the activity of JO-B and JO-C neurons were significantly correlated with both oscillation power and mean antennal angle, whereas in experiments using JO-AB-GAL4 and JO-CE-GAL4 drivers, correlations were only significant for mean antenna angle in JO-B neurons and only significant for oscillation power in JO-C neurons ( $p<0.01$ compared with EGFP controls, bootstrap analysis with Bonferroni correction). As with the results for the average flight responses, these subtle differences are likely due to slight variation in the GCaMP3 expression pattern in the GAL4 drivers. Although the responses to antennal motion clearly differed among classes of JO neurons, we also measured substantial variation across individuals. We tested whether this variability might be correlated with the absolute value of the antenna angle, oscillation power, or the average strength of the flight response but found no significant correlation with any of the parameters ( $p>0.05$, Pearson's correlation coefficient with Bonferroni correction).

Flies flying in the dark make slow antiphase and fast in-phase changes of stroke amplitude

Our previous behavioral study suggested that flies detect winginduced airflow through an antennal mechanosensory pathway and use it to decrease the amplitude of the contralateral wing, thereby enhancing the bilateral asymmetry in stroke amplitude (Mamiya et al., 2011). Results in Figure 3 show that JO-B, C, D, $\mathrm{EDM}$, and EVM neurons respond strongly to changes in the antennal oscillation power, suggesting that these cells might be responsible for regulating contralateral stroke amplitude. To test whether this was the case, we developed a paradigm for investigating how flies regulate stroke amplitude when they cannot rely on vision (Fig. 4A). In this setup, we tethered flies on the same holder used in the imaging studies but kept them without presentation of visual stimuli. The motivation for the paradigm was that, by removing the influence of visual-motor reflexes, which are very strong during flight, we could better isolate antennalmotor responses. We illuminated the flies from the back using an infrared LED and tracked the changes in the angular position of the wings at the ventral reversal point of the wing stroke (defined here as "stroke angle") using a machine vision system (Fig. 4A), and used it as a measure of stroke amplitude (Maimon et al., 2010). Figure $4 B$ shows an example time course of the left and right stroke angle for a fly flying in the dark. As can be seen in this example, the left and right stroke angles exhibit slow antiphasic oscillations such that, when one wing angle increased, the other stroke angle decreased (Fig. 4B, top row). In addition to this slow antiphase oscillation, we also observed smaller in-phase changes in stroke angle that occurred at higher frequencies (Fig. $4 B$, bottom row). To better quantify these two phenomena, we calculated the oscillation power of stroke angle at different frequencies and also estimated the coherence and phase lag between the stroke angles of the two wings (Fig. 4C). Oscillation power was larger at lower frequencies and decayed monotonically, whereas coherence was high at both low and high frequencies with a dip near 1-3 Hz. At lower frequencies, changes in the stroke angle of the two wings tended to be antiphase $(\sim \pm \pi)$, whereas at high frequency the changes were in-phase $(\sim 0)$. Thus, the spectral analysis in Figure $4 C$ confirms the qualitative impression of the trace in Figure $4 B$. 

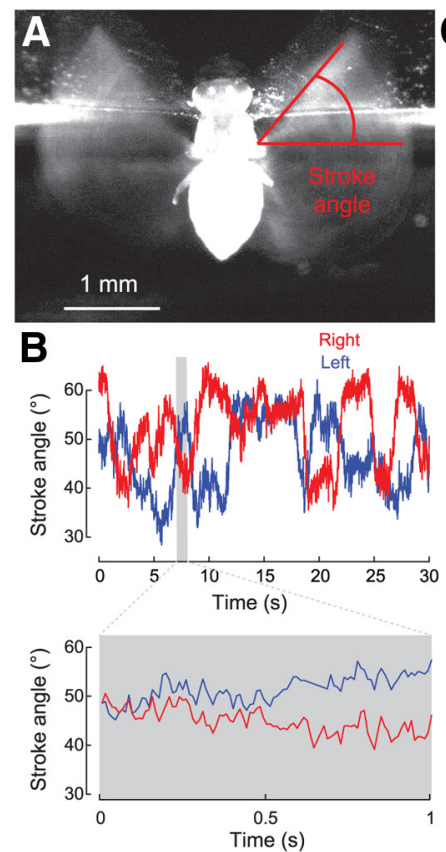
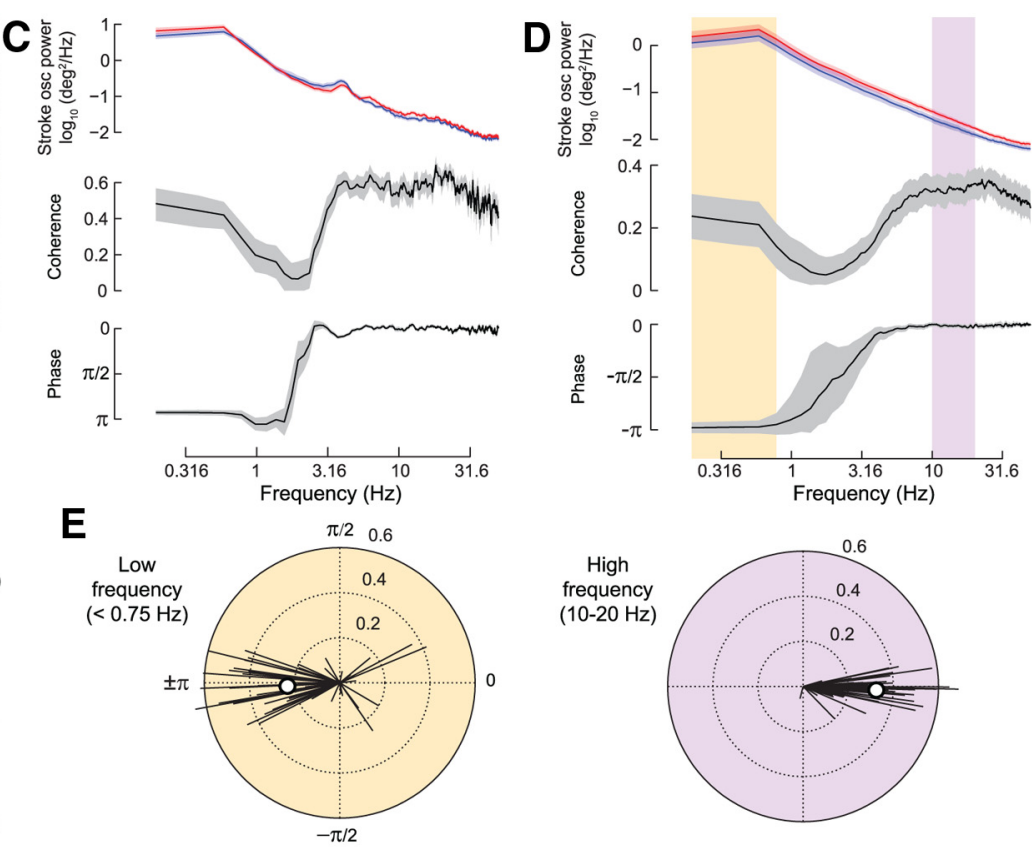

Figure 4. Tethered flies flying in the dark generate both large slow antiphase oscillations and small fast in-phase oscillations of stroke angles. $A$, A ventral view of a tethered flying fly. We defined stroke angle as an angle between the ventral flip point of the wing stroke envelope and the horizontal plane of the image. $\boldsymbol{B}$, Top, Example time course of the left (blue) and right (red) stroke angles during flight in the dark. Gray shaded box represents a region zoomed in on the bottom panel. Stroke angles show large slow antiphase oscillations. Bottom, Zoomed in view of a region indicated by the gray shaded box in the top panel indicating small fast in-phase oscillations of stroke angles. C, The power of the left (blue) and right (red) stroke angle oscillation (top), coherence (middle), and phase lag (bottom) between the two stroke angles at different frequencies during a 6 min flight in the dark. Gray shadings around the average lines represent $95 \%$ confidence intervals. The stroke angle oscillation power decreased as the frequency increased. This decrease was linear when the oscillation power and frequency were plotted on a log-log plot, suggesting scale-free distribution of the oscillation power. There were two peaks of coherence. At the low-frequency peak, the stroke angles were oscillating antiphase to each other; at the high-frequency peak, the stroke angles were oscillating in-phase. Positive phase lags indicate that the right stroke angle is phase advanced relative to the left stroke angle. To facilitate comparison with other phase-frequency plots, the vertical axis for this phase-frequency plot is reversed. $\boldsymbol{D}$, Same as $\boldsymbol{C}$ but for the average of 46 wild-type (CS) flies. The plot confirms that the results shown in $\boldsymbol{C}$ are consistent across flies. Orange and purple shaded boxes represent the low $(<0.75 \mathrm{~Hz})$ and high $(10-20 \mathrm{~Hz})$ frequency bands we selected for a closer inspection of the individual flies' behaviors. $\boldsymbol{E}$, Polar plots showing the coherence (radius) and the phase lag (angle) between the left and right stroke angles in the low-frequency $(<0.75 \mathrm{~Hz}$; left side, shaded orange) and high-frequency $(10-20 \mathrm{~Hz}$; right side, shaded purple) bands. Black lines indicate the coherence and phase lag for individual flies. White circles with black outline represent averages for each group.

Figure $4 D$ shows the results of our analysis averaged across 46 wild-type flies, demonstrating that these low-frequency antiphase oscillations of stroke angle and in-phase oscillations are very consistent. A dip in coherence near $1-3 \mathrm{~Hz}$ and a corresponding widening of the $95 \%$ confidence interval for the phase relationship between the two stroke angles were also consistent across the flies. The most parsimonious explanation for this dip in coherence is that there is a mechanism that operates at higher frequencies to keep two stroke angles in phase and another that operates at lower frequencies to keep them in antiphase, and that these two mechanisms interfere with each other at intermediate frequencies, resulting in a more variable relationship. We found that the log of stroke angle power decreased linearly with the log of frequency. This distribution of the oscillation power is suggestive of a power-law distribution $\left(P \propto 1 / f^{\beta}\right.$, where $P$ is power, $f$ is frequency, and $\beta$ is a parameter) that has been observed in many brain activities and behaviors that are arrhythmic (Voss and Clarke, 1975; Freeman and van Dijk, 1987; Gilden et al., 1995; Lowen et al., 1997; Zarahn et al., 1997). However, because similar power-law distributions can be generated by different types of mechanisms (He et al., 2010), further analyses and experiments are necessary before we can speculate on the neural mechanisms underlying this relationship.

To inspect each individual fly's behavior more closely, we plotted each value of coherence and phase lag measured within both a low $(<0.75 \mathrm{~Hz})$ and high $(10-20 \mathrm{~Hz})$ frequency band in polar coordinates (Fig. 4E). The resulting pair of plots (color coded throughout as orange and purple) illustrate the shift from antiphase to in-phase modulation of stroke angle at low and high frequency. These plots reveal that the coherence and phase lag are more variable at low frequency, suggesting that antiphase correlations may be influenced by the state of the animal during flight. It is important to emphasize that this phenomenon simply emerges from tethered flies flying in the dark. The experiments described next were designed to investigate the influence of JO neurons on this behavior.

\section{Genetic ablation of the JO-CE neurons disrupts the slow antiphase oscillation but not the fast in-phase oscillation of stroke angle}

If the JO-B, C, D, EDM, and EVM neurons are involved in generating the slow antiphase oscillation of stroke angle in the dark, then ablating these neurons should disrupt the phenomenon. We initially attempted to ablate all JO neurons by expressing the protein synthesis inhibitor ricin A (Landel et al., 1988) using an intersectional strategy combining JO1-GAL4 driver with the eyFLP tissue-specific recombination system (Newsome et al., 2000) (see Materials and Methods), but these flies were too behaviorally impaired to fly, probably due to ricin A expression in many cells in the eye in addition to the JO neurons. Therefore, we selectively expressed ricin $\mathrm{A}$ in either JO-AB or JO-CE neurons by combining more selective JO-AB-GAL4 or JO-CE-GAL4 drivers with eyFLP. This allowed us to test whether the selective ablation of JO-CE neurons, whose activity during flight showed stronger 

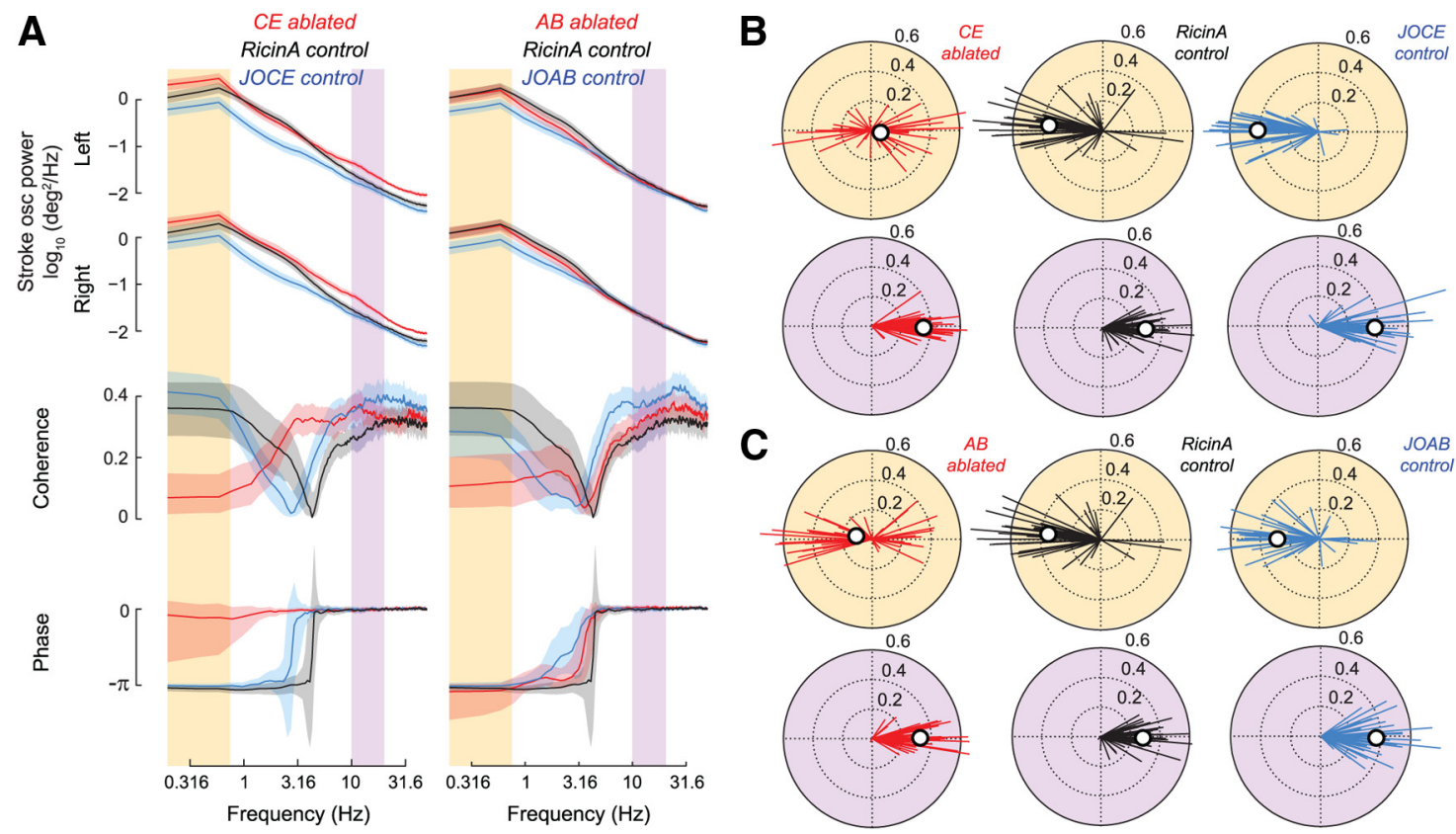

Figure 5. Genetically ablating $\mathrm{J} 0$ neurons disrupts the coherent antiphase oscillation of the stroke angles in the low-frequency band $(<0.75 \mathrm{~Hz})$. $A$, Left, The average power of the left and right stroke angle oscillation (top), average coherence (middle), and phase lag (bottom) between the two stroke angles at different frequencies for J0-CE ablated flies (red lines, $n=51$ ) and their parental control flies (RicinA control, black lines, $n=47 ; J 0$-CE control, blue lines, $n=47$ ) flying in the dark. Positive phase lags indicate that the right stroke angle is phase advanced relative to the left stroke angle. Right, The same as the plot on the left but for J0-AB ablated flies (red lines, $n=47$ ) and their parental control flies (RicinA control, black lines, $n=47 ; J 0-A B$ control, blue lines, $n=46$ ). Data for RicinA control flies are the same as those shown in the left panel but duplicated here to facilitate comparison with the experimental flies. Red, black, and blue shadings represent $95 \%$ confidence intervals for each plotted value. The orange and purple shaded boxes represent the low frequency $(<0.75 \mathrm{~Hz})$ and high-frequency $(10-20 \mathrm{~Hz})$ bands we selected for a detailed inspection of the individual flies' behavior in $B$ and $C$. Genetic ablation of JO-AB/JO-CE neurons lowered the coherence between the left and right stroke angles in the low-frequency band. J0-CE ablated flies exhibited an in-phase oscillation of stroke angles even in the low-frequency band. Genotype for CE ablated flies; eyFLP;JO-CE-GAL4 $\times$ UAS $\gg$ RicinA;UAS-2XEGFP, RicinA control flies; UAS $\gg R$ RicinA;UAS$2 \times E G F P \times C S, J O-C E$ control flies; eyFLP;JO-CE-GAL4 $\times C S$, AB ablated flies; eyFLP;JO-AB-GAL4 $\times$ UAS $\gg$ RicinA;UAS-2XEGFP, and JO-AB control flies; eyFLP;JO-AB-GAL4 $\times C S$. B, Polar plots showing the coherence (radius) and phase lag (angle) between the left and right stroke angles in the low-frequency $(<0.75 \mathrm{~Hz}$; left side, shaded orange) and high-frequency $(10-20 \mathrm{~Hz}$; right side, shaded purple) bands for individual J0-CE ablated flies (red lines) and their parental control flies (RicinA control, black lines; JO-CE control, blue lines). White circles with black outline represent averages. C, The same polar plots as B, but for JO-AB ablated flies (red lines) and their parental control flies (RicinA control, black lines; JO-AB control, blue lines). Data for RicinA control flies are the same as those shown in $\boldsymbol{B}$, but duplicated here to facilitate comparison with the experimental flies.

correlation with changes in antennal oscillation power compared with JO-B neurons, have a larger effect on the slow antiphase changes of stroke angle compared with the ablation of JO-AB neurons. In these experiments, the progeny of wild-type (CS) flies crossed to the relevant parental strains (JO-AB-/JO-CE-GAL4; eyFLP, or UAS $\gg$ RicinA) served as our controls. Genetic ablation of JO-CE neurons significantly decreased the mean coherence between left and right stroke angle in the low-frequency band $(<0.75 \mathrm{~Hz})$ and reduced the mean phase lag to near zero (Fig. $5 \mathrm{~A}$, left column middle and bottom row). In contrast, the coherence and phase lag in the higher frequency band $(10-20 \mathrm{~Hz})$ were not affected by the genetic ablation, suggesting that JO-CE neurons are not involved in generating the fast in-phase oscillation of stroke angle (Fig. 5A, left column middle and bottom row). Genetic ablation of JO-CE neurons also increased the power of wing stroke oscillation at both the low and high-frequency bands (Fig. $5 A$, left column top row; for both the left and right wings at both the low and high-frequency bands, $p<0.05$, Tukey-Kramer post hoc comparison of the means against parental control flies).

Closer inspection of the coherence and phase lag of individual flies in the low-frequency band $(<0.75 \mathrm{~Hz})$ reveals a fly-by-fly variation in our ablation experiment that complicates interpretation of population means (Fig. 5B, top row). For example, some of the JO-CE ablated flies exhibited antiphase oscillation of stroke angle, suggesting that JO-CE neurons are not the sole agents contributing to bilaterally asymmetric changes in wing motion at low frequencies. This is consistent with the possibility that JO-B and
JO-D neurons are also involved. Furthermore, because a small proportion of the control flies also showed slow synchronized oscillation of stroke angle (Fig. 5B, top row), the phase lag between the two wing angles at the lower frequencies likely depends on additional factors, such as the behavioral state of the individual fly. In contrast to the large individual variability in the lowfrequency band $(<0.75 \mathrm{~Hz})$, almost all the JO-CE ablated flies and the parental control flies showed highly coherent synchronized oscillation of the two wing angles in the higher frequency band ( $10-20 \mathrm{~Hz})$ (Fig. 5B, bottom row).

Consistent with the imaging results suggesting that $\mathrm{JO}-\mathrm{AB}$ neurons may play only a minor role in determining the correlation between the stroke angles of the two wings during flight, genetic ablation of JO-AB neurons had a much smaller effect on the stroke angle power, coherence, and phase (Fig. $5 \mathrm{~A}$, right column). Ablating JO-AB neurons did not increase the power of wing stroke oscillation significantly compared with the parental control flies (Fig. 5A, right column top row; for both the left and right wings at both the low- and high-frequency bands, $p>0.05$, Tukey-Kramer post hoc comparison of the means against parental control flies). In flies without JO-AB neurons, the population average of the coherence between stroke angle of the two wings decreased in the low-frequency band $(<0.75 \mathrm{~Hz})$ compared with the parental controls, but this decrease was smaller than in JO-CE ablated flies and the $95 \%$ confidence interval of the coherence overlapped with one of the parental controls (Fig. 5A, right column). Further, genetic ablation of JO-AB neurons did not change 

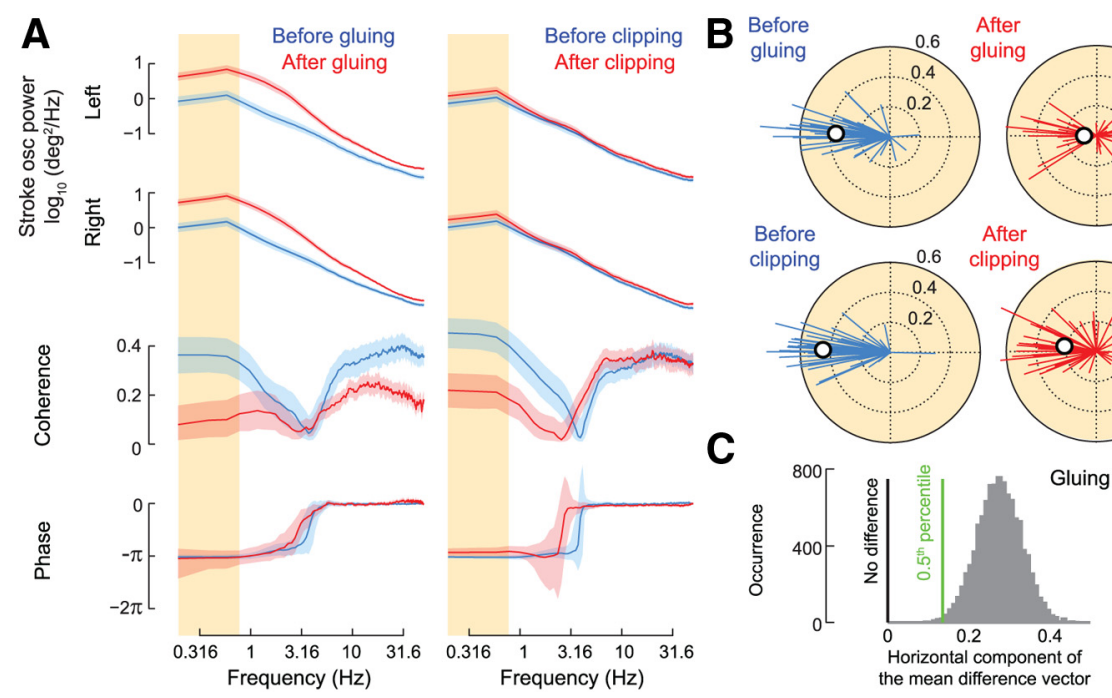

Figure 6. Physically reducing the inputs to JO neurons by gluing the second and third antennal segments together or clipping aristae mimics the effect of J0 neuron ablation. $A$, The average power of the left and right stroke angle oscillation (top), and the average coherence (middle) and phase lag (bottom) between the two stroke angles at different frequencies before (blue) and after (red) antennae gluing (left column, $n=44$ ), or aristae clipping (right column, $n=50$ ). Positive phase lags indicate that the right stroke angle is phase advanced relative to the left stroke angle. Red and blue shadings represent $95 \%$ confidence intervals for each plotted value. Orange shaded boxes represent the low-frequency band $(<0.75 \mathrm{~Hz})$ we selected for a detailed inspection of the individual fly's behaviors in $\boldsymbol{B}$. The antennae gluing and the aristae clipping both decreased the coherence of the antiphasic stroke angle oscillation in the low-frequency band, mimicking $\mathrm{J} 0 \mathrm{neuron}$ ablation. The phase is sign reversed after aristae clipping to facilitate comparison. Because there was no difference in the effect of physical manipulations among different groups of parental control flies (RicinA control, JO-AB control, and JO-CE control; for genotypes, see Fig. 5), we combined the results from all parental control flies in these plots. B, Polar plots showing the coherence (radius) and phase lag (angle) between the left and right stroke angles in the low-frequency band ( $<0.75 \mathrm{~Hz}$; shaded orange) for individual flies before (left column, blue lines) and after (center column, red lines) antennae gluing (top row), or aristae clipping (bottom row), and the difference vector between the two conditions (right column, black lines). White circles with black outline represent averages. Antennae gluing and aristae clipping increase the horizontal component in the coherence-phase polar plot, mimicking the effect of J0 neuron ablation. C, Distributions of the average horizontal component of the difference vectors calculated with bootstrap analysis $(n=10,000)$ for antennae gluing (left) or aristae clipping (right). Green vertical lines indicate the bottom 0.5 percentile of each distribution. Black lines indicate where the horizontal component is zero. For both antennae gluing and aristae clipping, the bottom 0.5 percentile line is located in the positive region, showing that the difference vectors have horizontal components that are significantly more positive than expected by chance at $\alpha=0.01$.

the average phase lag between the left and right wing angles at low or high frequency (Fig. $5 A$, right column bottom row).

Inspection of the behavior of individual flies at the lowfrequency band $(<0.75 \mathrm{~Hz})$ indicates that a larger proportion of flies with ablated $\mathrm{JO}-\mathrm{AB}$ neurons exhibited in-phase oscillation of stroke angle compared with the parental control flies (Fig. 5C, top row). However, this effect was less prominent compared with the flies with ablated JO-CE neurons. As was the case with flies without JO-CE neurons, the coherence and phase lag between the stroke angles of the two wings were less variable across individuals in the high-frequency band $(10-20 \mathrm{~Hz}$ ) (Fig. 5C, bottom row).

\section{Attenuation of JO sensitivity by physical manipulation mimics the effect of genetic ablation}

The results in Figure 5 suggest that JO-CE neurons, and to a lesser extent JO-AB neurons, are involved in determining the phase lag between the left and right stroke angle in low-frequency band $(<0.75 \mathrm{~Hz})$ during flight in the dark. As with many genetic ablation protocols, however, our experimental paradigm includes several caveats and it is possible that the changes we observed were not directly related to ablating JO neurons. For example, ricin A expression may have induced unexpected changes in the downstream circuits due to the lack of JO neurons during postlarval development. For this reason, we wanted to further confirm our results by directly interfering with JO function by gluing the joint between the third and second antennal segments or clipping the aristae. The aristae are rigidly connected to the third antennal segment and function as a lever that couples air movement to rotation of the third antennal segment relative to the second (Göpfert and Robert, 2001, 2002). Clipping the aristae thus attenuates mechanosensory transduction by JO neurons, as does gluing the second and third antennal segments together. These two methods have been used successfully in prior studies (Manning, 1967; von Schilcher, 1976; Budick et al., 2007; Duistermars et al., 2009; Kamikouchi et al., 2009; Yorozu et al., 2009; Fuller et al., 2014), but they do have limitations. Gluing the antenna may result in an artificial tonic input to JO neurons, and clipping the aristae does not completely impair antennal function as any shaft remaining after ablation (as well as the third antennal segment itself) may be sufficient to activate some JO neurons with air movements. Because of these limitations, these physical manipulations may have effects similar, but not identical, to genetic ablation. For both physical manipulations, different groups of parental control flies (Fig. 5 for genotypes) showed similar responses. Thus, we grouped together the results from all parental control flies in these experiments.

As shown in Figure $6 A$, both the antennae gluing and the aristae clipping increased the oscillation power of stroke angle at the low-frequency band, but this effect was larger for the antenna gluing (for the antennae gluing, $t_{(43)}=10.04$ and 9.90, $p<7.59 \times$ $10^{-13}$ and $p<1.17 \times 10^{-12}$ for the left and right wing, respectively; for the aristae clipping, $t_{(49)}=3.26$ and 3.07, $p<2.0 \times$ $10^{-3}$ and $<3.5 \times 10^{-3}$ for the left and right wing, respectively; paired $t$ test). Similar to the JO neuron ablation, both manipulations decreased the average coherence between the left and right stroke angle in the low-frequency band (Fig. 6A, middle row), although this effect was slightly smaller for the aristae clipping. However, in both physical manipulations, the average phase lag between the stroke angles of the two wings did not change significantly (Fig. 6A, bottom row). Inspection of data from individual flies in the low-frequency band suggests that gluing and clipping 
tend to decrease coherence for the antiphase oscillation of stroke angle and in some cases wings switch to in-phase oscillation (Fig. $6 B$, left and middle column). This effect is similar to the genetic ablation of JO-AB and JO-CE neurons. To further examine this effect, we calculated the difference vector for each manipulation (Fig. 6B, right column). Most of these vectors pointed to the right, confirming that antennae gluing and aristae clipping decreased antiphase oscillation and increased in-phase oscillation of stroke angle. Using bootstrap analysis, we assessed the likelihood of such a change occurring by chance and found that the effect is statistically significant for both manipulations (Fig. $6 C$, $p<0.01)$. In summary, gluing the antennae and clipping the aristae both mimic the changes in the coherence and phase lag caused by genetic ablation of JO neurons, although the magnitude of the effect is not as large.

\section{Flies without JO neurons can use visual feedback to produce antiphase oscillation of the wing stroke angle}

Results so far suggest that flies flying in the dark use JO neurons to detect the changes in the antennal movements caused by their flapping wings and use this information to modulate the left and right wing stroke angles in antiphase manner. In the final set of experiments, we asked whether flies need mechanosensory feedback through JO neurons to generate this antiphase modulation. We provided the flies with visual feedback using LED panels displaying wide-field vertical gratings whose horizontal movements were controlled by the difference between the left and right wing angles. In this standard closed-loop condition, when a fly tries to turn to the left by increasing the right stroke angle relative to the left, the visual pattern moves to the right, as if a fly is actually turning to the left in this virtual environment. To distinguish between the changes caused by the visual feedback from those caused by changes in brightness and the presentation of the visual pattern, we also presented static wide-field vertical stripes. We found that visual feedback increases oscillation power of the wing angle within a narrow frequency band centered around $2 \mathrm{~Hz}$ (Fig. $7 A$, left column, top row). This increase in the oscillation power was accompanied by a large increase in the coherence between the stroke angles in the same frequency band (Fig. $7 \mathrm{~A}$, left column, middle row). Phase-frequency plots indicate that the stroke angle of the two wings oscillate in antiphase manner within this frequency band (Fig. 7A, left column, bottom row). The presentation of a static pattern actually decreased oscillation power and did not increase the coherence at $2 \mathrm{~Hz}$, suggesting that the increase in the power and coherence of wing angles during closedloop visual feedback are not caused by the presentation of the visual pattern itself but rather are a result of the active feedback (Fig. 7A, left column). These results are not surprising, and are consistent with previous observations that flies adjust their wing stroke angles in a coherent antiphase manner to keep a vertical stripe in front of them during the standard closed-loop condition (Reichardt and Wenking, 1969; Götz, 1987). Coherent antiphasic oscillation of the wing stroke angles at $2 \mathrm{~Hz}$ suggests that flies are generating turning responses at this frequency. Previous studies on tethered flies flying in visual closed-loop have found that the frequency of turning responses peak near $3.5 \mathrm{~Hz}$ when a striped drum is used as a panorama (Wolf and Heisenberg, 1990) and peak near $1-2 \mathrm{~Hz}$ when a single stripe is used as a visual stimulus (Heisenberg and Wolf, 1979; Wolf and Heisenberg, 1990). These frequencies are close to the peak of the wing angle oscillation power that we found $(\sim 2 \mathrm{~Hz})$, and the slight shift in the peak frequency may be due to the differences in the details of the visual stimuli used, method of tethering, and how the turning responses were measured in these experiments (i.e., yaw torque vs wing stroke amplitude). Because the frequency and amplitude of these turning responses do not change much when the gain of the feedback is altered (Wolf and Heisenberg, 1990), they are thought to be actively generated by the fly rather than caused by the instability in the visual feedback loop. However, further experiments are necessary before we could have a better understanding of the mechanisms underlying the wing angle oscillation.

Inspection of each individual fly's behavior in a frequency band near $2 \mathrm{~Hz}$ shows that, when flies flew in the dark, coherence between the left and right stroke angles was small and the phase lag was highly variable, resulting in a small mean coherence vector (Fig. 7B, left column, top row). However, when the flies received visual feedback, coherence between the two stroke angles increased and the phase lag between the two wings became more narrowly distributed near $\pm \pi$, indicating antiphase modulation (Fig. 7B, middle column, top row). Taking the difference between the two conditions in a coherence-phase plane resulted in large vectors that pointed leftward (Fig. $7 B$, right column, top row). These difference vectors had horizontal components that were significantly more negative than those expected by chance (Fig. $7 C$, top row, $p<0.01$, bootstrap analysis), confirming that visual feedback indeed increases coherent antiphasic changes of the stroke angles around $2 \mathrm{~Hz}$.

Having characterized control flies, we next tested whether flies without JO-AB or JO-CE neurons can oscillate their left and right stroke angles in an antiphase manner in response to the visual feedback. Flies without JO-AB or JO-CE neurons still responded to visual feedback with antiphase oscillation of the two stroke angles (Fig. $7 \mathrm{~A}$, middle and right columns), suggesting that $\mathrm{JO}-\mathrm{AB}$ or JO-CE neurons are not necessary for flies to adjust stroke angles using visual feedback. Similar to the CS flies, flies lacking JO-AB or JO-CE neurons increased the oscillation power $\sim 2 \mathrm{~Hz}$ when they were under visual closed-loop conditions (Fig. $7 A$, middle and right columns, top rows). This increase in the oscillation power was accompanied by a large increase in the coherence between the two stroke angles in the same frequency band (Fig. 7A, middle and right columns, middle rows). Interestingly, this increase in the coherence was larger for the JO-AB/ JO-CE ablated flies compared with wild-type flies. Phasefrequency plots show that two wings oscillate antiphase with each other in this frequency band (Fig. 7A, middle and right columns, bottom rows).

Inspection of the individual flies' behavior at a frequency band near $2 \mathrm{~Hz}$ suggests that, in the dark, most of the flies lacking JO-AB neurons showed weak coherence between the two stroke angles, and they tended to oscillate them in antiphase (Fig. $7 B$, left column, middle row). Most of the flies lacking JO-CE neurons also showed weak coherence, but the wing stroke angles tended to oscillate in-phase (Fig. $7 B$, left column, bottom row). In both groups of flies, visual feedback of wing motion greatly increased coherence, and the phase lag was centered near $\pm \pi$, indicating antiphase modulation (Fig. $7 B$, middle column, middle and bottom rows). The resulting difference vectors pointed to the left (Fig. $7 B$, right column, middle and bottom rows), and the magnitude of the horizontal component was significantly more negative than expected by chance (Fig. $7 C$, middle and bottom rows; $p<0.01$, bootstrap analysis).

Results from these experiments suggest that, although JO neurons are involved in generating slow antiphase modulations of the left and right wing stroke angles when flies are flying in the dark, JO neurons are not necessary for antiphase oscillation of the wing stroke angles when visual feedback is available. Further- 
A

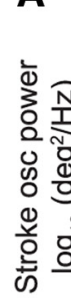

CS

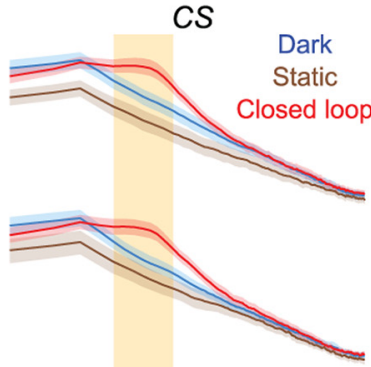

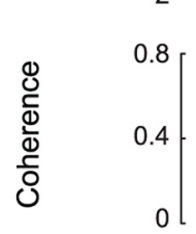

D
$\frac{⿹}{0}$
$\frac{C}{2}$

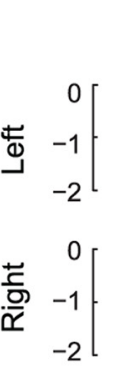

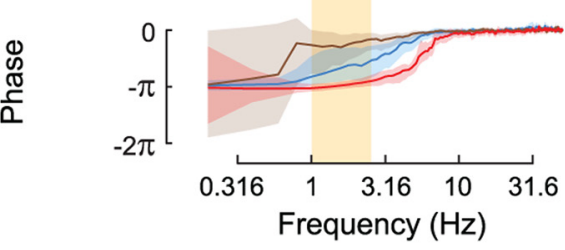

B Dark

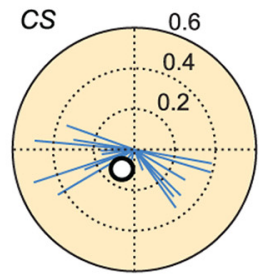

$A B$

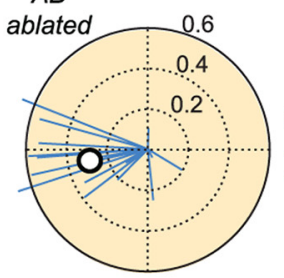

CE
Closed loop
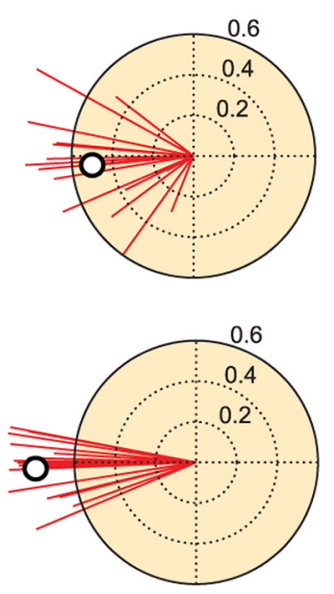

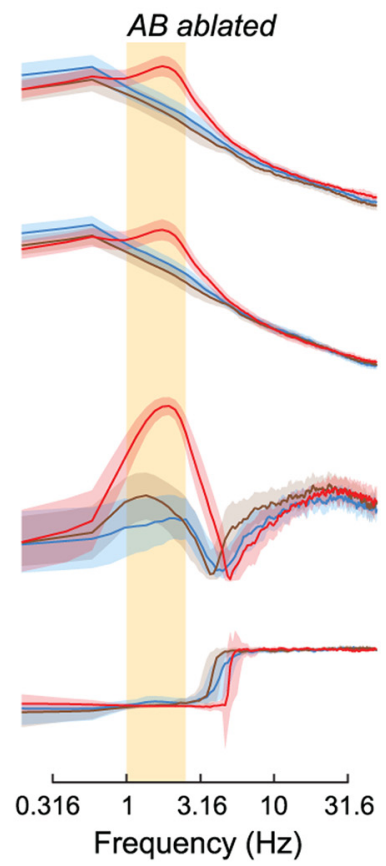

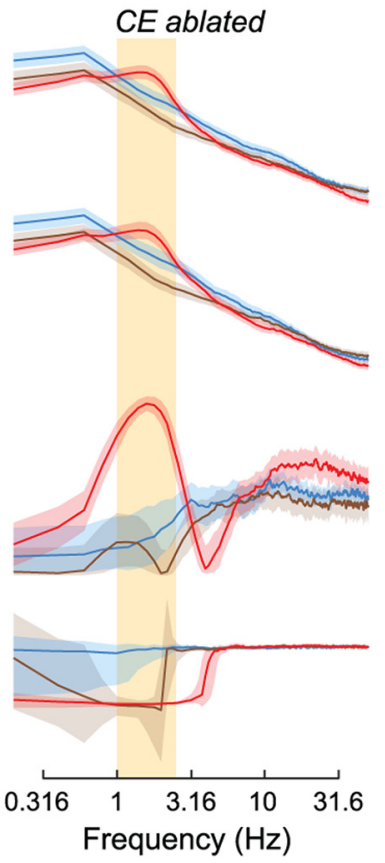
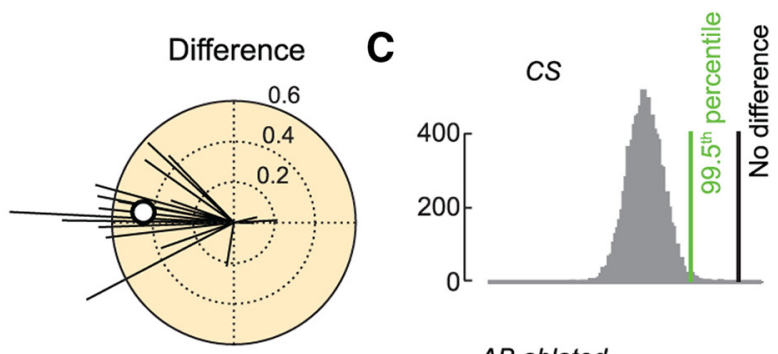

$A B$ ablated
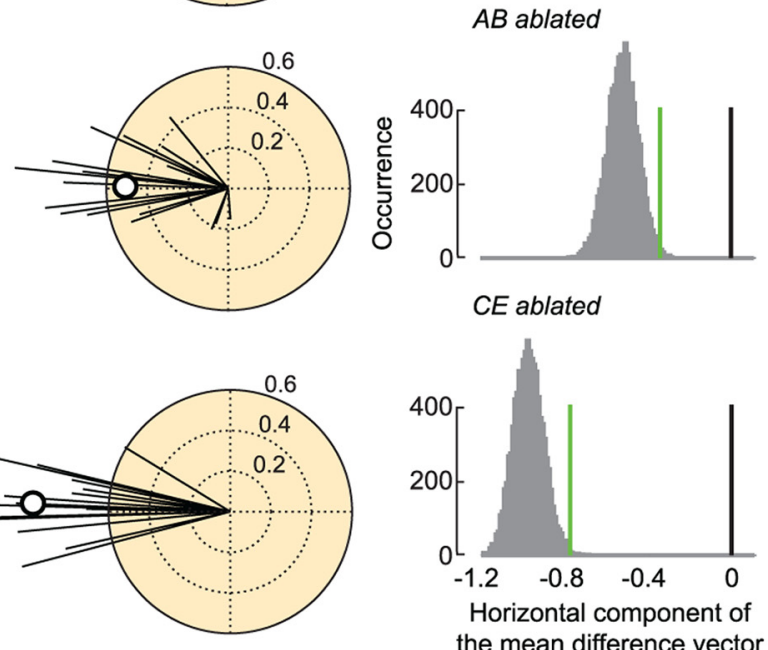

Figure 7. JO-AB/JO-CE ablated flies can still produce slow antiphase oscillation of stroke angles when visual feedback is provided. $A$, The average power of the left and right stroke angle oscillation (top), average coherence (middle), and phase lag (bottom) between the two stroke angles at different frequencies for wild-type flies (left column, $n=18$ ), J0-AB ablated flies (middle column, $n=$ 18), and J0-CE ablated flies (right column, $n=18$ ) flying in the dark (blue lines), with static visual stimuli (brown lines), or with closed-loop visual feedback of the stroke angles (red lines). Positive phase lags indicate that the right stroke angle is phase advanced relative to the left stroke angle. Blue, brown, and red shadings represent $95 \%$ confidence intervals for each plotted value. Orange shaded boxes show a frequency band (1-2.5 Hz) we selected for detailed inspection of the individual fly's behaviors in $\boldsymbol{B}$. In all groups of flies, closed-loop visual feedback increased the stroke angle oscillation power in a narrow frequency band near $2 \mathrm{~Hz}$. This increase in power was accompanied by an increase in coherence between the two stroke angles. Stroke angles oscillated antiphase to each other in this frequency band. The phase sign for AB ablated flies in closed loop is reversed to facilitate comparison. Genotype for AB ablated flies and C $E$ ablated flies is the same as in Figure 5. $\boldsymbol{B}$, Polar plots showing the coherence (radius) and phase lag (angle) between the left and right stroke angles in the frequency band indicated by orange boxes in $\boldsymbol{A}(1-2.5 \mathrm{~Hz}$ ) for individual wild-type flies (top row), J0-AB ablated flies (middle row), or J0-CE ablated flies (bottom row) flying in the dark (left column), with closed-loop visual feedback (middle column), and the difference vector between the two conditions (right column). White circles with black outline represent averages. In all groups, providing closed-loop visual feedback increased the coherence toward antiphasic oscillation of the stroke angles. C, Distributions of the average horizontal component of the difference vectors calculated with bootstrap analysis $(n=10,000)$ for wild-type flies (top row), J0-AB ablated flies (middle row), and J0-CE ablated flies (bottom row). Green vertical lines indicate the 99.5th percentile of the distributions. Black lines indicate the position for the zero horizontal component. For all group of flies, the 99.5 th percentile line is located in the negative region, showing that the difference vectors have horizontal components that are significantly more negative than expected by chance at $\alpha=0.01$. 


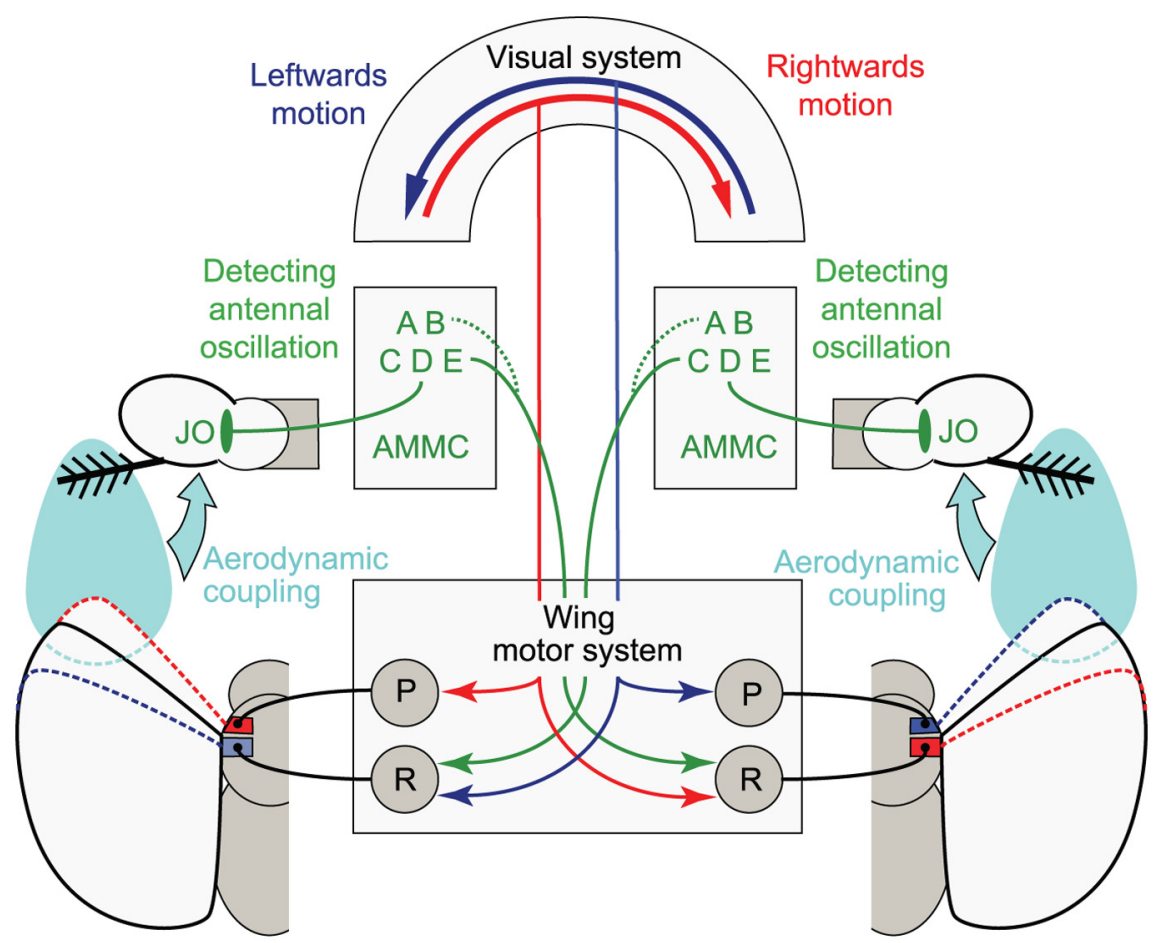

Figure 8. A schematic model of how J0 neurons may control the phase relationship between the left and right wing stroke angles. J0 neurons detect the magnitude of the antennal oscillations caused by the wing-induced airflow. When the ipsilateral wing moves closer to the antenna and the antenna oscillation power increases, J0 neuron activity increases. J0-C, D, E neurons respond stronger to these increases compared with JO-A and JO-B neurons. Hypothetical descending interneurons in the AMMC that are postsynaptic to these $\mathrm{J} 0$ neurons send signals to the wing motor system to reduce the stroke angle of the contralateral wing. In tethered flies flying without visual feedback, this J0-mediated response results in a low-frequency $(<0.75 \mathrm{~Hz})$ antiphase oscillation of the two stroke angles. Visual feedback (detection of the leftwards and rightwards motion) can also produce this antiphase relationship between the two stroke angles through a pathway that does not require J0 neurons. P, Promoter; R, remoter: activities in the wing motor system that result in the increase or decrease of the wing stroke angle, respectively.

more, the fact that flies lacking JO-A, B or JO-C, E neurons can oscillate their wing stroke angles in an antiphase manner when flying in visual-closed loop conditions provides further evidence that the disruption of antiphase oscillation of wing stroke angle we observed in the dark is not due to general defects in the flight control system.

\section{Discussion}

Our imaging results show that all JO neuron terminals, except those in the EDM branch, increase their activity in response to the ipsilateral antennal motion during flight (Fig. 2). With the exception of JO-A neurons, this activity is strongly correlated with the power of antennal oscillation at the wing beat frequency (Fig. 3). Genetically ablating JO neurons disrupts the slow antiphasic modulation of wing stroke angle exhibited by flies flying in the dark (Figs. 4, 5), and this effect can be mimicked by physical manipulations of the antennae that reduce JO neuron sensitivity (Fig. 6). Collectively, these results are consistent with a model in which flying flies use multiple JO neuron classes to detect the increase in oscillatory airflow that occurs when the stroke amplitude of the ipsilateral wing increases, and the JO neurons are involved in reducing the stroke angle of the contralateral wing (Fig. 8) (Mamiya et al., 2011). It is still not clear how the changes in JO neuron activity affect the contralateral wing angle (Fig. 8), but future analyses of downstream neural circuits in flying flies by recording, activation, and silencing may provide a better understanding. It is noteworthy that flies missing JO neurons are still capable of exhibiting antiphasic modulation of wing stroke angles under visual closed-loop conditions (Fig. 7), indicating that the JO neurons are not required for this flight behavior. This suggests that the role of JO neurons in maintaining this coordination becomes important when visual feedback is absent.

Although previous studies have suggested that JO-C, D, E neurons respond better to tonic antennal movements than to phasic movements (Kamikouchi et al., 2009; Yorozu et al., 2009), we found that during flight they respond strongly to antennal oscillation but not to tonic changes in the antenna angle (Fig. $3 B$ ). There may be several reasons for this apparent discrepancy. First, during tethered flight, the magnitude of the antennal oscillation can be very large (aristae-tip displacements of 2-15 $\mu \mathrm{m}$ ), whereas the tonic deflection induced by wing motion $(1-7 \mu \mathrm{m})$ is much smaller than the typical stimuli used in previous studies (Mamiya et al., 2011). The activation of JO-C, D, E neurons by the tonic antennal deflection may become larger when flies experience strong winds, or airflow generated by self-motion during free-flight. Second, the antennal oscillation induced by wing motion during flight is closer to a sawtooth pattern (A.M., unpublished data) than to a pure sinusoidal oscillation that has been used in previous studies, and the JO-C, D, E neurons may be more sensitive to higher harmonics. Third, flies raise their antennae forward during flight (Burkhardt and Gewecke, 1965), shifting the baseline angle between the third and second antennal segments relative to the resting position on which previous studies were performed. Because insect chordotonal organs can alter their response properties based on their baseline position (Hofmann et al., 1985; Matheson, 1990, 1992), this change in position during flight may have increased the sensitivity to phasic oscillations. Fourth, it is possible that the JO neuron's response properties may have changed with flight, similar to what has been shown for visual interneurons (Maimon et al., 2010; Jung et al., 2011; Suver et al., 2012; Schnell et al., 2014; Tuthill et al., 2014) and central complex neurons (Seelig and Jayaraman, 2013; Weir et al., 2014). In locusts, the sensitivity of a wing stretch receptor is increased during flight due to a release of octopamine by dorsal unpaired median cells (Ramirez and Orchard, 1990). Finally, studies of wing campaniform neurons indicate that mechanoreceptors that respond tonically to step deformations can nevertheless follow oscillatory stimuli at high frequency (Dickinson and Palka, 1987). A recent study has also suggested that JO-D neurons can respond to both tonic and phasic stimuli (Matsuo et al., 2014).

JO-A neurons were the only antennal mechanosensory neurons whose activity during flight did not correlate with the power of antennal oscillation (Fig. $3 B$ ). This might indicate that the JO-A neurons are saturated at the large oscillation amplitudes (2-15 $\mu \mathrm{m})$ that occur during tethered flight (Mamiya et al., 2011). A previous study has shown that responses of JO-A neurons tend to saturate when the oscillation magnitude exceeds 5 $\mu \mathrm{m}$ (Kamikouchi et al., 2009), which is consistent with this hy- 
pothesis. Another possibility is that the JO-A neurons habituate quickly to the large oscillatory stimuli. Consistent with this hypothesis, the response of JO-A neurons to large tonic antennal displacements has been shown to adapt quickly (Yorozu et al., 2009) and we found that typical JO-A neurons respond strongly at the beginning of the flight and gradually decrease its activity (see, e.g., Fig. 1D).

Results from our behavioral studies suggest that a response involving JO neurons reduces the contralateral wing stroke angle and contributes to the antiphasic changes of the left and right wing stroke amplitude (Figs. 5, 6). However, this antiphasic wing coordination was only evident in the low-frequency band $(<0.75$ $\mathrm{Hz}$ ) (Figs. 4, 5, 6). There are several nonexclusive explanations for the absence of the response involving JO neurons at higher frequencies. One possibility is that the dynamics of the response are too slow to generate the coordination at high frequency. Another possibility is that JO neuron activity may need to increase by a certain amount to activate the response, and this level of increase is only achieved during low-frequency oscillation when the changes in the wing stroke angles are sufficiently large (Fig. 4B). Finally, there could be another neural mechanism that synchronizes the wing stroke amplitudes at higher frequencies, and this mechanism may overwrite the response involving JO neurons. This hypothesis is consistent with the results from behavioral experiments showing that wing stroke amplitudes change synchronously at higher frequencies and that these synchronous movements are not affected by JO neuron ablations (Fig. 5) or physical manipulations of the antennae (Fig. 6). Although tethered flight preparations allow us to control sensory stimuli and perform physiological experiments, they impose restrictions that make it difficult to determine the behavioral role of the response involving JO neurons during free flight. Nevertheless, our results suggest several possibilities. The response we observed seems to enhance the asymmetry between the stroke amplitude of the two wings (Fig. 5). Assuming that the difference in wing stroke amplitude correlates with yaw torque (Götz et al., 1979; Tammero et al., 2004), roll (Dickinson, 1999; Sherman and Dickinson, 2003; Muijres et al., 2014), or sideslip (Sugiura and Dickinson, 2009), this response may be a part of a mechanism that initiates (Duistermars et al., 2009; Duistermars and Frye, 2010) or increases the magnitude (Mamiya et al., 2011) of a turn or a sideslip maneuver. Alternatively, the response may maintain the large bilateral differences in wing stroke amplitude that are necessary to compensate for an intrinsic bias, such as one caused by wing damage. Regardless of the function, as visual feedback was sufficient to generate large bilaterally asymmetric changes in wing stroke angle (Fig. 7), this response may only become important when visual feedback is weak, unreliable, saturated, or habituated. These situations may occur when flies are flying high above ground, in very dim conditions, or when flies are flying sideways during casting maneuvers (van Breugel and Dickinson, 2014). If the response involving JO neurons acts at a slow time scale as suggested by tethered flight experiments in the dark (Figs. 4, 5, 6), the time course of the response fits better with functions such as continuously generating sideslip and compensating for wing damage than those that involve rapid maneuvers. Because the effects of antennal manipulations on turning behavior seem to depend on behavioral context (Budick et al., 2007; Duistermars et al., 2009) and type of visual stimuli presented (Mamiya et al., 2011), the response involving JO neurons may have different functions depending on the situation as well.

The present study is consistent with a model in which flying flies integrate inputs from JO-B, C, D, and E neurons to detect an increase in wing-induced airflow and initiate a response that reduces the contralateral wing stroke angle (Fig. 8). By using multiple JO neuron classes with different sensitivity, flies may be able to detect a wide range of changes in wing-induced airflow. This may become important during free flight when changes in wing stroke amplitude tend to be much smaller than those observed during tethered flight (Fry et al., 2003; Bergou et al., 2010; Muijres et al., 2014). Matsuo et al. (2014) have recently identified a descending interneuron called AMMC D1, which has dendritic arbors that overlap with axon terminals of JO-B, C, D, and E neurons and projects its axon to the thorax. Future recordings from this and other descending interneurons originating from the AMMC (Kamikouchi et al., 2009) in tethered flying flies may allow us to study how flies integrate the activities of multiple JO neuron classes with other sensory inputs to control wing motion in a context appropriate way.

\section{References}

Bergou AJ, Ristroph L, Guckenheimer J, Cohen I, Wang ZJ (2010) Fruit flies modulate passive wing pitching to generate in-flight turns. Phys Rev Lett 104:148101. CrossRef Medline

Borst A (2014) Fly visual course control: behaviour, algorithms and circuits. Nat Rev Neurosci 15:590-599. CrossRef Medline

Budick SA, Reiser MB, Dickinson MH (2007) The role of visual and mechanosensory cues in structuring forward flight in Drosophila melanogaster. J Exp Biol 210:4092-4103. CrossRef Medline

Burkhardt D, Gewecke M (1965) Mechanoreception in Arthropoda: the chain from stimulus to behavioral pattern. Cold Spring Harb Symp Quant Biol 30:601-614. CrossRef Medline

Dickinson MH (1999) Haltere-mediated equilibrium reflexes of the fruit fly, Drosophila melanogaster. Philos Trans R Soc Lond B Biol Sci 354:903916. CrossRef Medline

Dickinson MH, Palka J (1987) Physiological-properties, time of development, and central projection are correlated in the wing mechanoreceptors of Drosophila. J Neurosci 7:4201-4208. Medline

Duistermars BJ, Frye MA (2010) Multisensory integration for odor tracking by flying Drosophila: behavior, circuits and speculation. Commun Integr Biol 3:60-63. CrossRef Medline

Duistermars BJ, Chow DM, Frye MA (2009) Flies require bilateral sensory input to track odor gradients in flight. Curr Biol 19:1301-1307. CrossRef Medline

Eberl DF, Hardy RW, Kernan MJ (2000) Genetically similar transduction mechanisms for touch and hearing in Drosophila. J Neurosci 20:59815988. Medline

Freeman WJ, van Dijk BW (1987) Spatial patterns of visual cortical fast EEG during conditioned reflex in a rhesus monkey. Brain Res 422:267-276. CrossRef Medline

Fry SN, Sayaman R, Dickinson MH (2003) The aerodynamics of free-flight maneuvers in Drosophila. Science 300:495-498. CrossRef Medline

Fuller SB, Straw AD, Peek MY, Murray RM, Dickinson MH (2014) Flying Drosophila stabilize their vision-based velocity controller by sensing wind with their antennae. Proc Natl Acad Sci U S A 111:E1182-E1191. CrossRef Medline

Gewecke M (1970) Antennae: another wind sensitive receptor in locusts. Nature 225:1263-1264. CrossRef Medline

Gewecke M, Heinzel HG, Philippe J (1974) Role of antennae of dragonfly Orthetrum-cancellatum in flight control. Nature 249:584-585. CrossRef

Gilden DL, Thornton T, Mallon MW (1995) 1/f noise in human cognition. Science 267:1837-1839. CrossRef Medline

Göpfert MC, Robert D (2001) Biomechanics: turning the key on Drosophila audition. Nature 411:908. CrossRef Medline

Göpfert MC, Robert D (2002) The mechanical basis of Drosophila audition. J Exp Biol 205:1199-1208. Medline

Götz KG (1987) Course-control, metabolism and wing interference during ultralong tethered flight in Drosophila melanogaster. J Exp Biol 128:35-46.

Götz KG, Hengstenberg B, Biesinger R (1979) Optomotor control of wing beat and body posture in Drosophila. Biol Cybern 35:101-112. CrossRef

Guizar-Sicairos M, Thurman ST, Fienup JR (2008) Efficient subpixel image registration algorithms. Opt Lett 33:156-158. CrossRef Medline

Halfon MS, Gisselbrecht S, Lu J, Estrada B, Keshishian H, Michelson AM 
(2002) New fluorescent protein reporters for use with the Drosophila Gal4 expression system and for vital detection of balancer chromosomes. Genesis 34:135-138. CrossRef Medline

He BJ, Zempel JM, Snyder AZ, Raichle ME (2010) The temporal structures and functional significance of scale-free brain activity. Neuron 66:353369. CrossRef Medline

Heisenberg M, Wolf R (1979) On the fine structure of yaw torque in visual flight orientation of Drosophila melanogaster. J Comp Physiol 130:113130. CrossRef

Heran H (1957) Die Bienenantenne Als Messorgan Der Flugeigengeschwindigkeit. Naturwissenschaften 44:475. CrossRef

Hofmann T, Koch UT, Bassler U (1985) Physiology of the femoral chordotonal organ in the stick insect, Cuniculina-Impigra. J Exp Biol 114:207-223.

Hollick FSJ (1940) The flight of the dipterous fly Muscina stabulans fallén. Philo Trans R Soc Lond B Biol Sci 230:357-390. CrossRef

Johnston C (1855) Auditory apparatus of the Culex mosquito. Q J Microscop Sci 3:97-102.

Jung SN, Borst A, Haag J (2011) Flight activity alters velocity tuning of fly motion-sensitive neurons. J Neurosci 31:9231-9237. CrossRef Medline

Kamikouchi A, Shimada T, Ito K (2006) Comprehensive classification of the auditory sensory projections in the brain of the fruit fly Drosophila melanogaster. J Comp Neurol 499:317-356. CrossRef Medline

Kamikouchi A, Inagaki HK, Effertz T, Hendrich O, Fiala A, Göpfert MC, Ito K (2009) The neural basis of Drosophila gravity-sensing and hearing. Nature 458:165-171. CrossRef Medline

Kim J, Chung YD, Park DY, Choi S, Shin DW, Soh H, Lee HW, Son W, Yim J, Park CS, Kernan MJ, Kim C (2003) A TRPV family ion channel required for hearing in Drosophila. Nature 424:81-84. CrossRef Medline

Landel CP, Zhao J, Bok D, Evans GA (1988) Lens-specific expression of recombinant ricin induces developmental defects in the eyes of transgenic mice. Genes Dev 2:1168-1178. CrossRef Medline

Lowen SB, Cash SS, Poo M, Teich MC (1997) Quantal neurotransmitter secretion rate exhibits fractal behavior. J Neurosci 17:5666-5677. Medline

Maimon G, Straw AD, Dickinson MH (2010) Active flight increases the gain of visual motion processing in Drosophila. Nat Neurosci 13:393-399. CrossRef Medline

Mamiya A, Straw AD, Tómasson E, Dickinson MH (2011) Active and passive antennal movements during visually guided steering in flying Drosophila. J Neurosci 31:6900-6914. CrossRef Medline

Manning A (1967) Antennae and sexual receptivity in Drosophila melanogaster females. Science 158:136-137. CrossRef Medline

Matheson T (1990) Responses and locations of neurons in the locust metathoracic femoral chordotonal organ. J Comp Physiol A Neuroethol Sens Neural Behav Physiol 166:915-927.

Matheson T (1992) Range fractionation in the locust metathoracic femoral chordotonal organ. J Comp Physiol A Neuroethol Sens Neural Behav Physiol 170:509-520.

Matsuo E, Yamada D, Ishikawa Y, Asai T, Ishimoto H, Kamikouchi A (2014) Identification of novel vibration- and deflection-sensitive neuronal subgroups in Johnston's organ of the fruit fly. Front Physiol 5:179. CrossRef Medline

Mitra P, Bokil H (2008) Observed brain dynamics. New York: Oxford UP.

Muijres FT, Elzinga MJ, Melis JM, Dickinson MH (2014) Flies evade looming targets by executing rapid visually directed banked turns. Science 344:172-177. CrossRef Medline

Newsome TP, Asling B, Dickson BJ (2000) Analysis of Drosophila photoreceptor axon guidance in eye-specific mosaics. Development 127:851-860. Medline

Niehaus M (1981) Flight and flight control by the antennae in the small tortoiseshell (Aglais-urticae L-lepidoptera): 2. Flight mill and free flight experiments. J Comp Physiol 145:257-264. CrossRef

Olsen SR, Bhandawat V, Wilson RI (2007) Excitatory interactions between olfactory processing channels in the Drosophila antennal lobe. Neuron 54:89-103. CrossRef Medline
Ramirez JM, Orchard I (1990) Octopaminergic modulation of the forewing stretch-receptor in the locust Locusta-Migratoria. J Exp Biol 149:255-279.

Reichardt W, Wenking H (1969) Optical detection and fixation of objects by fixed flying flies. Naturwissenschaften 56:424-425. CrossRef Medline

Reiser MB, Dickinson MH (2008) A modular display system for insect behavioral neuroscience. J Neurosci Methods 167:127-139. CrossRef Medline

Sane SP, Dieudonné A, Willis MA, Daniel TL (2007) Antennal mechanosensors mediate flight control in moths. Science 315:863-866. CrossRef Medline

Schnell B, Weir PT, Roth E, Fairhall AL, Dickinson MH (2014) Cellular mechanisms for integral feedback in visually guided behavior. Proc Natl Acad Sci U S A 111:5700-5705. CrossRef Medline

Seelig JD, Jayaraman V (2013) Feature detection and orientation tuning in the Drosophila central complex. Nature 503:262-266. CrossRef Medline

Sherman A, Dickinson MH (2003) A comparison of visual and halteremediated equilibrium reflexes in the fruit fly Drosophila melanogaster. J Exp Biol 206:295-302. CrossRef Medline

Straw AD, Dickinson MH (2009) Motmot, an open-source toolkit for realtime video acquisition and analysis. Source Code Biol Med 4:5. CrossRef Medline

Sugiura H, Dickinson MH (2009) The generation of forces and moments during visual-evoked steering maneuvers in flying Drosophila. PLoS One 4:e4883. CrossRef Medline

Sun XR, Badura A, Pacheco DA, Lynch LA, Schneider ER, Taylor MP, Hogue IB, Enquist LW, Murthy M, Wang SS (2013) Fast GCaMPs for improved tracking of neuronal activity. Nat Commun 4:2170. CrossRef Medline

Suver MP, Mamiya A, Dickinson MH (2012) Octopamine neurons mediate flight-induced modulation of visual processing in Drosophila. Curr Biol 22:2294-2302. CrossRef Medline

Tammero LF, Frye MA, Dickinson MH (2004) Spatial organization of visuomotor reflexes in Drosophila. J Exp Biol 207:113-122. CrossRef Medline

Taylor GK, Krapp HG (2008) Sensory systems and flight stability: what do insects measure and why? In: Advances in insect physiology: insect mechanics and control, pp 231-316. San Diego: Elsevier Academic.

Tian L, Hires SA, Mao T, Huber D, Chiappe ME, Chalasani SH, Petreanu L, Akerboom J, McKinney SA, Schreiter ER, Bargmann CI, Jayaraman V, Svoboda K, Looger LL (2009) Imaging neural activity in worms, flies and mice with improved GCaMP calcium indicators. Nat Methods 6:875881. CrossRef Medline

Tuthill JC, Nern A, Rubin GM, Reiser MB (2014) Wide-field feedback neurons dynamically tune early visual processing. Neuron 82:887-895. CrossRef Medline

van Breugel F, Dickinson MH (2014) Plume-tracking behavior of flying Drosophila emerges from a set of distinct sensory-motor reflexes. Curr Biol 24:274-286. CrossRef Medline

von Schilcher F (1976) The role of auditory stimuli in the courtship of Drosophila melanogaster. Anim Behav 24:18-26. CrossRef

Voss RF, Clarke J (1975) 1/f noise in music and speech. Nature 258:317-318. CrossRef

Weir PT, Schnell B, Dickinson MH (2014) Central complex neurons exhibit behaviorally gated responses to visual motion in Drosophila. J Neurophysiol 111:62-71. CrossRef Medline

Wolf R, Heisenberg M (1990) Visual control of straight flight in Drosophila melanogaster. J Comp Physiol A Neuroethol Sens Neural Behav Physiol 167:269-283. Medline

Yorozu S, Wong A, Fischer BJ, Dankert H, Kernan MJ, Kamikouchi A, Ito K, Anderson DJ (2009) Distinct sensory representations of wind and nearfield sound in the Drosophila brain. Nature 458:201-205. CrossRef Medline

Yule GU (1896) On the significance of Bravais' formulae for regression in the case of skew correlation. Proc R Soc Lond 60:477-489.

Zarahn E, Aguirre GK, D’Esposito M (1997) Empirical analyses of BOLD fMRI statistics: I. Spatially unsmoothed data collected under nullhypothesis conditions. Neuroimage 5:179-197. CrossRef Medline 